\title{
A comparison of refinement indicators for $p$-adaptive discontinuous Galerkin methods for the Euler and Navier-Stokes equations
}

\author{
Fabio Naddei * and Marta de la Llave Plata ${ }^{\dagger}$ and Vincent Couaillier ${ }^{*}$ \\ ONERA the French aerospace lab, Châtillon, 92322, France
}

\begin{abstract}
This paper presents an analysis of refinement indicators for the numerical solution of the Euler and the Navier-Stokes equations using a $p$-adaptive discontinuous Galerkin (DG) method. Residual error, discretization error and feature-based indicators are studied in the context of $\boldsymbol{p}$-adaptive DG simulations of two inviscid flow configurations, namely, the flow over a Gaussian bump and the flow past a circular cylinder. As expected, the superiority of local $p$-refinement as compared to uniform $p$-refinement is clearly observed. The results obtained highlight the efficiency of the considered refinement indicators with respect to an indicator based on the local entropy error. In particular, they identify regions where numerical errors are being produced and not those where errors are being convected. Finally the $p$-adaptive algorithm is applied to the simulation of the laminar flow past a circular cylinder at $\mathrm{Re}=40$. The great potential of the spectral decay ${ }^{1}$ and $^{V_{M}} \mathrm{MS}^{2}$ indicators, which combine accuracy and computational efficiency, is also demonstrated.
\end{abstract}

\section{Introduction}

High-order discontinuous Galerkin (DG) methods ${ }^{3-6}$ have been gaining growing interest among the scientific community in recent years. ${ }^{7,8}$ The DG methods are based on the variational projection of the Navier-Stokes equations and combine some features of Finite Volume (FV) and Finite Element (FE) methods. Remarkable properties of DG methods include the possibility of achieving arbitrarily high-order of accuracy, even at physical boundaries and on unstructured meshes, and high parallel efficiency on multi-core architectures, thanks to the compact stencil of the numerical scheme, each element requiring only information from direct neighbours. ${ }^{9}$

These properties play a fundamental role in the simulation of turbulent flows for which the finest scales of turbulence must be accurately resolved over long integration periods thus requiring efficient numerical methods with low dispersion and dissipation errors.

A large reduction in the computational cost of such simulations could be achieved by exploiting the possibility of increasing the local spatial resolution of DG methods by local mesh refinement ( $h$-refinement), and by locally increasing the polynomial order within the elements ( $p$-refinement). Local $p$-refinement can reduce dissipation and dispersion errors in regions where the solution is smooth by employing high polynomial degrees, thus allowing for the accurate resolution of unsteady turbulent phenomena with a lower number of degrees of freedom (DOF) as compared to Finite Difference (FD) and FV methods. ${ }^{10,11}$ On the other hand, $h$-refinement can isolate regions characterized by geometrical and physical discontinuities.

$h p$-adaptive techniques are based on the definition of refinement indicators and an $h p$-decision algorithm. Refinement indicators, usually relying on a posteriori error estimates, identify regions where an increased spatial resolution is required. The $h p$-decision procedure, on the other hand, measures the level of smoothness of the solution and selects the refinement strategy ( $h$ or $p$ ). Considerable efforts have been dedicated to the development of robust a posteriori error estimators for partial differential equations. We refer to the works by Roy, ${ }^{12}$ Houston and Süli, ${ }^{13}$ and Mitchell and McClain, ${ }^{14}$ for a review of common error estimation strategies and their use for $h p$-adaptive algorithms.

In this context three main classes of refinement indicators can be identified:

Feature based - this class of refinement indicators is based on physical or theoretical properties or on the observation that some phenomena must be fully resolved in order to obtain an accurate representation of the flow

*Ph.D. student, ONERA, Numerical Methods Group, École Polytechnique, fabio.naddei@onera.fr

${ }^{\dagger}$ Senior Research Engineer, ONERA, Numerical Methods Group, marta.de_la_llave_plata@onera.fr

${ }^{\ddagger}$ Group leader, ONERA, Numerical Methods Group, vincent.couaillier@onera.fr, AIAA Senior member. 
field or the computation of quantities of interest. Examples of feature-based refinement indicators include measures of vorticity or vortex detection methods, ${ }^{15}$ boundary layer detection, or those based on gradients of the mass fraction for two-phase flows. ${ }^{16}$ These methods are often inefficient and they might lack of robustness, as they do not take into account error propagation and the refinement of certain features might not be relevant for the computation of target quantities. However, their implementation is often simple and inexpensive and can provide reasonable results when employed by expert users.

Discretization Error (DE) based - The DE is defined as the difference between the numerical and the analytical solution. As the goal of a numerical simulation is to obtain a reliable approximation of the latter, adaptive refinement algorithms can be defined by identifying and refining areas where the DE is high. The most common strategy to estimate DE is to perform two simulations on a given mesh using incremental polynomial spaces or successively refined meshes and comparing the two numerical solutions. ${ }^{17}$ Other possible approaches include the computation of estimates of higher-order solutions, ${ }^{18}$ estimates of truncation error through the decay rate of Legendre expansion coefficients ${ }^{19}$ or the exploitation of superconvergent phenomena. ${ }^{20}$ The drawback of DE based algorithms is that the DE is produced in regions of insufficient spatial resolution and then diffused and convected as a scalar quantity. ${ }^{12,21}$ An adaptation process which is driven by local values of DE would refine also in regions where the DE is transported and not actually produced thus exhibiting sub-optimal performances. Residual Error (RE) based and $\tau$-based (TE) methods are often employed respectively in the FE and FV frameworks. This is due to the fact that TE and RE appear as production terms in the Discretization Error Transport equations. ${ }^{22-27}$ In the FE framework RE-based methods can often be classified as DE-based estimator since RE estimates can provide DE bounds provided that suitable norms are employed.

Goal Oriented - Goal Oriented techniques have been shown to be optimal for the definition of refinement indicators when a target quantity is the goal of a CFD simulation (e. g. the drag or the lift coefficient). That is to say, they provide the lowest computational cost to achieve a prescribed level of accuracy in the measure of the target functional. ${ }^{24,28,29}$ Their optimality reflects their ability to consider the inherent mechanism of error propagation in hyperbolic and nearly-hyperbolic problems. Their derivation requires the computation of element-wise residuals associated with the discrete solution. These residuals must be weighted by the solution of an adjoint problem derived from the discretization employed and the target quantity. One possible drawback is the sensitivity of such methods to the target selected. Moreover the solution of the adjoint problem must be performed in a refined polynomial space and requires the backward integration in time for unsteady problems, thus requiring the storage of the solution at each previous time step. Given the high computational cost of these operations, adjoint methods are not commonly used for unsteady configurations.

There exists a vast literature dedicated to the analysis of error indicators and refinement techniques. However, given the large variety of indicators introduced to date, the selection of such an indicator in the implementation of a new adaptive algorithm can be a daunting task. The aim of this research is to provide guidelines for the design of adaptive refinement algorithms with a particular focus on their suitability for unsteady turbulent flow simulations.

As previously mentioned, goal-oriented refinement indicators have been shown to be vastly superior to other error estimators for steady problems. Unfortunately, their high computational cost impedes their application to LES and DNS of turbulent flows. For this reason, adjoint-based methods are not considered in this work. On the other hand DE and feature-based indicators have already been successfully applied to LES. ${ }^{1,15,30,31}$ However, to the best of the authors' knowledge, there have been no attempts to extend and assess the efficiency of RE-based indicators for LES/DNS.

This analysis marks the beginning of an extended comparison of different refinement indicators. Here, we will assess the performance of DE, RE and feature-based refinement indicators in guiding adaptive $p$-refinement for the solution of the steady Euler and the Navier-Stokes equations using a DG method based on a modal approach. We focus on their ability to reduce the computational cost necessary to achieve a prescribed level of accuracy as well as potential implementation issues. We do not compare the accuracy of different refinement indicators on the evaluation of the exact error (usually measured by the effectivity index $i_{\text {eff }}=\frac{\text { error estimate }}{\text { exact error }}$ ) as different error norms are employed for different indicators.

This paper is organised as follows. In section II the considered equations and the DG method are briefly introduced. In section III a brief review of the considered refinement indicators is presented. A short introduction is provided along with their relationship with other refinement indicators employed in the literature. In section IV the direct comparison of refinement indicators on inviscid and viscous flows is provided. In section $\mathrm{V}$ we present our observations about possible implementation issues and the computational cost of the considered indicators. While the latter is of limited 
interest when considering steady flows, it will be of primary importance when using these indicators for unsteady flows computations. The main conclusions from this research are presented in section VI.

\section{Model problem and discretization}

The simulations presented in this work have been performed using the DG unstructured solver Aghora developed at ONERA $^{32}$ solving the compressible Navier-Stokes equations. The physical model and the DG method used in this work are recalled in this section.

\section{II.A. Physical model}

Let $\Omega \in \mathbb{R}^{d}$ be a bounded domain, where $d$ is the space dimension, the compressible Navier-Stokes equations take the form :

$$
\partial_{t} \mathbf{u}+\nabla \cdot \mathscr{F}_{c}(\mathbf{u})-\nabla \cdot \mathscr{F}_{v}(\mathbf{u}, \nabla \mathbf{u})=0
$$

with associated initial conditions $\mathbf{u}(\cdot, 0)=\mathbf{u}_{0}(\cdot)$ and appropriate boundary conditions prescribed on $\partial \Omega$. The vector $\mathbf{u}=(\rho, \rho \mathbf{v}, \rho E)^{T}$ represents the conservative state variables, with $\rho$ being the density, $\mathbf{v}=\left(U_{1}, U_{2}, U_{3}\right)^{T}$ the velocity vector and $E=\frac{p}{(\gamma-1) \rho}+\frac{\mathbf{v} \cdot \mathbf{v}}{2}$ the total specific energy. Here, $p$ is the static pressure and $\gamma=\frac{C_{p}}{C_{v}}>1$ the ratio of specific heats. The nonlinear convective fluxes and the diffusive fluxes in Eq. (1) are defined as:

$$
\mathscr{F}_{c, i}(\mathbf{u})=\left[\begin{array}{c}
\rho U_{i} \\
\rho U_{1} U_{i}+p \delta_{i 1} \\
\rho U_{2} U_{i}+p \delta_{i 2} \\
\rho U_{3} U_{i}+p \delta_{i 3} \\
\rho U_{i} E
\end{array}\right] \quad \mathscr{F}_{v, i}(\mathbf{u}, \nabla \mathbf{u})=\left[\begin{array}{c}
0 \\
\tau_{i 1} \\
\tau_{i 2} \\
\tau_{i 3} \\
\tau_{i k} U_{k}-q_{i}
\end{array}\right]
$$

with

$$
\begin{aligned}
\tau_{i j} & =\mu\left(\frac{\partial U_{j}}{\partial x_{i}}+\frac{\partial U_{i}}{\partial x_{j}}-\frac{2}{3} \frac{\partial U_{k}}{\partial x_{k}} \delta_{i j}\right) \\
q_{i} & =-\lambda \frac{\partial T}{\partial x_{i}} \\
T & =\frac{1}{C_{v}}\left(E-\frac{1}{2} U_{i} U_{i}\right)
\end{aligned}
$$

where $\mu$ is the dynamic viscosity, described by the Sutherland law, $T$ denotes the temperature, $\lambda=\mu \frac{C_{p}}{\operatorname{Pr}}$ is the thermal conductivity and Pr refers to the Prandtl number.

\section{II.B. The DG method}

The domain $\Omega$ is partitioned in a shape-regular mesh $\mathscr{T}_{k}$ consisting of $N$ non-overlapping and non-empty elements $K$ of characteristic size $h_{K}$. We also define the sets $\mathscr{E}_{i}$ and $\mathscr{E}_{b}$ of interior and boundary faces in $\mathscr{T}_{k}$ such that $\mathscr{E}_{h}=\mathscr{E}_{i} \cup \mathscr{E}_{b}$.

Let $S_{h}^{p}=\left\{\phi \in L^{2}(\Omega):\left.\phi\right|_{\mathscr{K}} \in \mathscr{P}^{p}(K), \forall K \in \mathscr{T}_{k}\right\}$ be the functional space formed by piecewise polynomials of either total or partial degree at most $p$ defined in the element, and $\left(\phi_{K}^{1}, \ldots \phi_{K}^{N_{p}}\right) \in \mathscr{P} p(K)$ a hierarchical and orthonormal basis of $\mathscr{P}^{p}(K)$ of dimension $N_{p}$. The polynomial order $p$ will in general depend on the element and will be indicated as $p_{K}$ when necessary. For the DG method used in this research, the orthonormal basis is obtained by applying a modified Gram-Schmidt procedure to a tensor product of monomials of degree at most $p$. This produces a diagonal mass matrix even on curved elements. ${ }^{33}$ The solution in each element is thus expressed as

$$
\mathbf{u}_{h}(\mathbf{x}, t)=\sum_{l=1}^{N_{p}} \phi_{K}^{l}(\mathbf{x}) \mathbf{u}_{K}^{l}(t), \quad \forall \mathbf{x} \in K, K \in \mathscr{T}_{k}, \forall t>0
$$

in which the polynomial coefficients $\left(\mathbf{u}_{k}^{l}\right)_{1 \leq l \leq N_{p}}$ represent the DOFs of the discrete problem in element $K$. The semidiscrete variational form of Eq. (1) therefore reads: find $\mathbf{u}_{h}$ in $S_{h}^{p}$ such that $\forall \phi_{h} \in S_{h}^{p}$

$$
\int_{\mathscr{T}_{K}} \phi_{h} \partial_{t} \mathbf{u}_{h} \mathrm{~d} V+\mathscr{L}_{c}\left(\mathbf{u}_{h}, \phi_{h}\right)+\mathscr{L}_{v}\left(\mathbf{u}_{h}, \phi_{h}\right)=0
$$


In Eq. (7) $\mathscr{L}_{c}$ and $\mathscr{L}_{v}$ represent the variational projection of the convective and viscous terms onto the functional space $S_{h}^{p}$. For a given interface $e$ in $\mathscr{E}_{i}$ we define the average operator $\left\{\{u\}=\left(u^{+}+u^{-}\right) / 2\right.$ and the jump operator $[[\mathbf{u}]]=\mathbf{u}^{+} \otimes \mathbf{n}-\mathbf{u}^{-} \otimes \mathbf{n}$, where $\mathbf{n}$ is the local outward unit normal. The DG discretization of the convective terms then reads

$$
\mathscr{L}_{c}\left(\mathbf{u}_{h}, \phi_{h}\right)=-\int_{\mathscr{T}_{K}} \mathscr{F}_{c}\left(\mathbf{u}_{h}\right) \cdot \nabla_{h} \phi_{h} \mathrm{~d} V+\int_{\mathscr{E}_{i}}\left[\left[\phi_{h}\right]\right] H_{c}\left(\mathbf{u}_{h}^{+}, \mathbf{u}_{h}^{-}, \mathbf{n}\right) \mathrm{d} S+\int_{\mathscr{E}_{b}} \phi_{h}^{+} \mathscr{F}_{c}\left(\mathbf{u}_{b}\right) \cdot \mathbf{n} \mathrm{d} S
$$

The boundary values $\mathbf{u}_{b}=\mathbf{u}_{b}\left(\mathbf{u}_{h}^{+}, \mathbf{u}_{\text {ext }}, \mathbf{n}\right)$, with $\mathbf{u}_{\text {ext }}$ a reference external state, are computed in order to impose the appropriate boundary condition on each boundary in $\mathscr{E}_{b}$.

The function $H_{c}$ is a numerical flux that approximates the convective flux on an element face and must be chosen to be conservative and consistent. In the simulations presented in this work the local Lax-Friedrichs (LLF) flux and the Roe flux have been employed. For the discretization of the viscous term in Eq. (7) the BR2 method of Bassi and coworkers $^{34,35}$ was employed, namely:

$$
\begin{aligned}
\mathscr{L}_{v}\left(\mathbf{u}_{h}, \phi_{h}\right)=\int_{\mathscr{T}_{K}} \mathscr{F}_{v}\left(\mathbf{u}_{h}, \nabla_{h} \mathbf{u}_{h}+\boldsymbol{L}_{h}\right) \cdot \nabla \phi_{h} \mathrm{~d} V-\int_{\mathscr{E}_{i}}\left[\left[\phi_{h}\right]\right]\left\{\left\{\mathscr{F}_{v}\left(\mathbf{u}_{h}, \nabla_{h} \mathbf{u}_{h}+\eta_{e} \boldsymbol{l}_{h}^{e}\right)\right\}\right] \cdot \mathbf{n d} S & \\
& -\int_{\mathscr{E}_{b}} \phi_{h}^{+} \mathscr{F}_{v}\left(\mathbf{u}_{b}, \nabla \mathbf{u}_{b}+\eta_{e} \boldsymbol{l}_{h}^{e}\right) \cdot \mathbf{n} \mathrm{d} S
\end{aligned}
$$

where $\eta_{e}$ is a numerical parameter that ensures the stability of the method. The global lifting operator $\boldsymbol{L}_{h}$ is defined as the sum of the local lifting operators $\boldsymbol{l}_{h}^{e}: \boldsymbol{L}_{h}=\sum_{e \in \partial K} \boldsymbol{l}_{h}^{e}$ where $\boldsymbol{l}_{h}^{e}$ has support on the elements adjacent to $e \in \mathscr{E}_{i}$ and is obtained from the solution of the following problem on the internal faces:

$$
\left.\int_{K^{+} \cup K^{-}} \phi_{h} \boldsymbol{l}_{h}^{e} \mathrm{~d} V=-\int_{e}\left\{\left\{\phi_{h}\right\}\right]\left[\mathbf{u}_{h}\right]\right] \mathrm{d} S \quad \forall \phi_{h} \in S_{h}^{p}
$$

A similar expression for the local lifting coefficients, consistent with the boundary conditions, is obtained on $\mathscr{E}_{b}$.

The semi-discrete equation Eq. (7) is discretized in time by means of the implicit backward-Euler scheme relying on the GMRES method and ILU(0) preconditioning for the solution of the corresponding linear system. The integrals in Eq. (7) are computed by means of the Gauss-Legendre quadrature with $q=p+1+m$ points in each space direction, where $m$ denotes the number of overintegration points used for dealiasing purposes.

\section{Refinement and marking procedure}

An adaptive $p$-refinement algorithm defines locally the polynomial degree $p_{K}$ used to approximate the solution within a given element. As mentioned in section I, in this work only stationary configurations are considered and the refinement procedure acts as follows. First a converged steady solution is obtained for a given refinement level. A global refinement indicator $\eta_{\text {glob }}$ is then computed in order to estimate the accuracy of the obtained solution, as an example the total entropy variation in an inviscid uniform flow. This quantity is then compared to a user-defined threshold $\omega$ specifying the accuracy level desired. Refinement is therefore deemed necessary if $\eta_{\text {glob }}>\omega$. In this case, element-wise refinement indicators $\eta_{K}$ are computed and used to identify elements where spatial resolution must be increased.

In this work, we use the maximum marking criterion. Namely, an element $K$ is marked for refinement if :

$$
\eta_{K}>\theta \max _{L \in \mathscr{T}_{K}} \eta_{L}
$$

where $\theta \in[0,1]$ is a user-defined marking parameter. In this work we set $\theta=0.5$. Moreover, we have decided to limit to 1 the maximum change in polynomial degree between two neighbouring elements. For this reason additional elements may be marked for refinement.

Once the marking is completed, a new set of $p$ values is computed by increasing by one the polynomial degree in marked elements. A maximum local polynomial degree is enforced to avoid instability issues and the need of excessive memory storage. The simulation is then restarted from the previous solution based on this the new set of $p$ values.

\section{III.A. Refinement Indicators}

In this section we present the refinement indicators considered in this research. First, three refinement indicators that can be classified as DE- or feature-based will be presented: the VMS indicator, the spectral decay indicator and the 
nonconformity error indicator. Then, two more indicators will be introduced: the residual-based and residuum-ncf based indicator. These can be described as RE-based indicators.

\section{III.A.1. The VMS indicator}

The first refinement indicator that we consider in this work was developed by Kuru et al. ${ }^{2}$ in the context of the variational multiscale simulation (VMS) approach. ${ }^{36}$ In the VMS framework the numerical solution vector $\mathbf{u}_{h}$ is expressed as:

$$
\mathbf{u}_{h}=\mathbf{u}_{h, L}+\mathbf{u}_{h, S}
$$

where $\mathbf{u}_{h, L}$ and $\mathbf{u}_{h, S}$ are the contributions to the numerical solution corresponding to the large-resolved and the smallresolved scales. In their work, the authors define $\mathbf{u}_{h, L} \in S_{h}^{p_{L}}$ and $\mathbf{u}_{h, S} \in S_{h}^{p} \backslash S_{h}^{p_{L}}$ being $0 \leq p_{L}<p$ the polynomial degree that defines the cut-off between large and small scales.

The refinement indicator proposed by Kuru et al. is then defined as the $L^{2}$-norm of the momentum density of the small-resolved scales:

$$
\eta_{K}:=\left(\int_{K}\left\|(\rho \mathbf{v})_{h, S}\right\|^{2} \mathrm{~d} V\right)^{\frac{1}{2}} \quad \eta_{g l o b}:=\left(\sum_{K} \eta_{K}^{2}\right)^{\frac{1}{2}}
$$

This refinement indicator was therefore interpreted as an approximation of the DE associated to the coarse-scale solution measured by the $L^{2}$-norm of the momentum density $\left\|(\rho \mathbf{v})_{h, L}-(\rho \mathbf{v})\right\|_{L^{2}(\Omega)}$.

We define the VMS refinement indicator as:

$$
\eta_{K}:=\left(\int_{K}\left\|(\rho \mathbf{v})_{h, p}-(\rho \mathbf{v})_{h, p-1}\right\|^{2} \mathrm{~d} V\right)^{\frac{1}{2}}
$$

where $(\rho \mathbf{v})_{h, p-1}$ is obtained from the projection of the numerical solution on $S_{h}^{p-1}$. This refinement indicator acts as a DE estimate for $\mathbf{u}_{h, p}$ employing a lower-order solution. It can also be interpreted as a feature-based refinement indicator, measuring the 'energy' of the highest-order modes.

This estimator bears strong similarities with the error indicator developed by Mavriplis ${ }^{19,37}$ where an additional term depending on the decay rate of polynomial coefficients is also included. This decay rate is helpful in identifying underresolved regions of the flow. However, the corresponding DE estimate has been shown to produce relatively low effectivity index values when considering two- and three-dimensional problems. ${ }^{38}$ For this reason, the refinement indicator derived by Mavriplis is not considered in this work.

\section{III.A.2. The spectral decay indicator}

The so-called spectral decay indicator was first introduced as a discontinuity sensor by Persson and Peraire ${ }^{39}$ for shock capturing in order to stabilize numerical simulations in the presence of discontinuities. The spectral decay indicator, also known as smoothness indicator, is defined as:

$$
S_{e}:=\frac{\left\|f\left(\mathbf{u}_{h, p}\right)-f\left(\mathbf{u}_{h, p-1}\right)\right\|_{L^{2}(K)}}{\left\|f\left(\mathbf{u}_{h, p}\right)\right\|_{L^{2}(K)}}
$$

where again $\mathbf{u}_{h, p-1}$ represents the projection of the numerical solution on $S_{h}^{p-1}$ and $f$ is a function that depends on the solution. Persson and Peraire employed this smoothness indicator as a shock capturing parameter based on either the entropy or the enthalpy for $f(\mathbf{u})$. Gassner et al. ${ }^{40}$ considered Eq. (15) based on the momentum density in one direction and employed this estimator as a refinement indicator. Tumolo et al. ${ }^{1,31,41,42}$ used this refinement indicator for the simulation of various CFD configurations. Following their approach, we define the spectral decay indicator as:

$$
\eta_{K}:=\frac{\left\|(\rho \mathbf{v})_{h, p}-(\rho \mathbf{v})_{h, p-1}\right\|_{L^{2}(K)}}{\left\|(\rho \mathbf{v})_{h, p}\right\|_{L^{2}(K)}}
$$

Equation (16) corresponds to the error estimator provided in Eq. (14) normalized by the total 'energy' of the cell defined by $\left\|(\rho \mathbf{v})_{h, p}\right\|_{L^{2}(K)}$. 


\section{III.A.3. The nonconformity error indicator}

The third refinement indicator considered in this work is the so-called nonconformity (NCF) error. This indicator is based on the assumption that, unless a physical discontinuity is present, the analytical solution is continuous across element faces. This implies that a jump in the numerical solution can be considered as a a measure of the error. Krivodonova et al. ${ }^{43,44}$ introduced the following discontinuity sensor:

$$
\eta_{K}:=\max _{\mathscr{E} \in \partial K} \max _{j}\left|\frac{\left.\left[u_{h}\left(x_{j}\right)\right]\right]}{2\left\{\left\{u_{h}\left(x_{j}\right)\right\}\right.}\right|
$$

where $u_{h}$ is a variable like density or pressure and $x_{j}$ are the quadrature points on the element faces. This error estimator can also be considered as a local DE estimator thanks to the superconvergent phenomena at outflow boundaries for some DG methods. ${ }^{43,44}$ Gassner and Altmann ${ }^{40}$ employed this quantity as a refinement indicator. Following their approach, we indicate as nonconformity error indicator:

$$
\eta_{K}:=\max _{\mathscr{E} \in \partial K} \max _{j} \frac{\left\|\left(\rho \mathbf{v}_{h}\right)^{+}\left(x_{j}\right)-\left(\rho \mathbf{v}_{h}\right)^{-}\left(x_{j}\right)\right\|}{2\left\|\left\{\left(\rho \mathbf{v}_{h}\right)\left(x_{j}\right)\right\}\right\|}
$$

\section{III.A.4. The residual-based indicator}

The residual-based refinement indicator that will be described here follows the classical derivation of residual-based error estimators for FE method ${ }^{45}$ and the derivation by Hartmann and Houston. ${ }^{46}$ Let us write the semidiscrete form of Eq. (7) as:

$$
\mathscr{N}_{h}\left(\mathbf{u}_{h}, \phi\right)=0 \quad \forall \phi \in S_{h}^{p}
$$

and let $J(\mathbf{u})$ be a nonlinear target functional. Provided that the target functional satisfies a compatibility condition and the discretized operator is adjoint consistent, ${ }^{24}$ it is possible to show that:

$$
J(\mathbf{u})-J\left(\mathbf{u}_{h}\right)=-\mathscr{N}_{h}\left(\mathbf{u}_{h}, z\right)
$$

where $z$ is the solution of an adjoint problem derived from the target functional $J(\cdot)$. The derivation of this result corresponds to the general derivation of duality-based a posteriori error estimators. Manipulating the semidiscrete operator, it is possible to obtain:

$$
\mathscr{N}_{h}\left(\mathbf{u}_{h}, \phi\right)=\sum_{K \in \mathscr{T}_{K}}\left[\int_{K} \mathbf{R}\left(\mathbf{u}_{h}\right) \phi \mathrm{d} V+\int_{\partial K \cap \mathscr{E}_{i}} \mathbf{r}\left(\mathbf{u}_{h}\right) \phi^{+} \mathrm{d} S+\int_{\partial K \cap \mathscr{E}_{b}} \mathbf{r}_{b}\left(\mathbf{u}_{h}\right) \phi^{+} \mathrm{d} S\right]
$$

in which the terms on the right-hand side are defined as:

$$
\begin{aligned}
\mathbf{R}\left(\mathbf{u}_{h}\right) & =\frac{\partial \mathbf{u}_{h}}{\partial t}+\nabla \cdot \mathscr{F}_{c}\left(\mathbf{u}_{h}\right)-\nabla \cdot \mathscr{F}_{v}\left(\mathbf{u}_{h}, \nabla_{h} \mathbf{u}_{h}+\boldsymbol{L}_{h}\right) \\
\mathbf{r}\left(\mathbf{u}_{h}\right) & =\left[H_{c}\left(\mathbf{u}_{h}^{+}, \mathbf{u}_{h}^{-}, \mathbf{n}\right)-\mathscr{F}_{c}\left(\mathbf{u}_{h}^{+}\right) \cdot \mathbf{n}\right]-\left[\left\{\left[\mathscr{F}_{v}\left(\mathbf{u}_{h}, \nabla_{h} \mathbf{u}_{h}+\eta_{e} r_{h}^{e}\right)\right\}\right]-\mathscr{F}_{v}\left(\mathbf{u}_{h}^{+}, \nabla_{h} \mathbf{u}_{h}^{+}+\boldsymbol{L}_{h}\right)\right] \cdot \mathbf{n} \\
\mathbf{r}_{b}\left(\mathbf{u}_{h}\right) & =\left[\mathscr{F}_{c}\left(\mathbf{u}_{b}\right)-\mathscr{F}_{c}\left(\mathbf{u}_{h}^{+}\right)\right] \cdot \mathbf{n}-\left[\mathscr{F}_{v}\left(\mathbf{u}_{b}, \nabla_{h} \mathbf{u}_{b}+\eta_{e} r_{h}^{e}\right)-\mathscr{F}_{v}\left(\mathbf{u}_{h}^{+}, \nabla_{h} \mathbf{u}_{h}^{+}+\boldsymbol{L}_{h}\right)\right] \cdot \mathbf{n}
\end{aligned}
$$

Where the internal residual Eq. (22) measures how accurately the numerical solution within an element satisfies the continuous mathematical model, the interelement residuals Eq. (23) measures the jump in normal fluxes at internal faces and the boundary residual Eq. (24) measures the error in the imposition of boundary conditions.

Provided that the adjoint solution is $\left.z\right|_{K} \in H^{s}(K)$ with $2 \leq s \leq p_{K}+1, \forall K \in \mathscr{T}_{K}$, it can be shown ${ }^{24}$ that the error in the target functional can be bounded as

$$
\left|J(\mathbf{u})-J\left(\mathbf{u}_{h}\right)\right| \leq C_{\text {int }}\left(\sum_{K \in \mathscr{T}_{K}}\left(\eta_{K}\right)^{2}\right)^{\frac{1}{2}}
$$

where $C_{\text {int }}$ is a positive constant which depends on the shape-regularity of the mesh, the polynomial degree $p$ and the regularity of the dual solution, and

$$
\eta_{K}:=h_{K}^{s}\left\|\mathbf{R}\left(\mathbf{u}_{h}\right)\right\|_{L^{2}(K)}+h_{K}^{s-\frac{1}{2}}\left\|\mathbf{r}\left(\mathbf{u}_{h}\right)\right\|_{L^{2}\left(\partial K \cap \mathscr{E}_{i}\right)}+h_{K}^{s-\frac{1}{2}}\left\|\mathbf{r}_{b}\left(\mathbf{u}_{h}\right)\right\|_{L^{2}\left(\partial K \cap \mathscr{E}_{b}\right)}
$$


On the basis of numerical experiments, Hartmann and Houston employed therefore the following residual-based inticator:

$$
\eta_{K}:=h_{K}\left\|\mathbf{R}\left(\mathbf{u}_{h}\right)\right\|_{L^{2}(K)}+h_{K}^{\frac{1}{2}}\left\|\mathbf{r}\left(\mathbf{u}_{h}\right)\right\|_{L^{2}\left(\partial K \cap \mathscr{E}_{i}\right)}+h_{K}^{\frac{1}{2}}\left\|\mathbf{r}_{b}\left(\mathbf{u}_{h}\right)\right\|_{L^{2}\left(\partial K \cap \mathscr{E}_{b}\right)} \quad \eta_{\mathrm{glob}}:=\left(\sum_{K \in \mathscr{T}_{K}}\left(\eta_{K}\right)^{2}\right)^{\frac{1}{2}}
$$

Rivière and Wheeler ${ }^{26}$ derived a similar error estimator for an elliptic problem, with linear diffusion and production, by employing the energy norm of the operator in place of the $L^{2}$-norm.

\section{III.A.5. The residuum-NCF based indicator}

Dolejš i $^{47}$ proposed a refinement indicator based on the following global error definition:

$$
E:=\left(R\left(\mathbf{u}_{h}\right)^{2}+\operatorname{NCF}\left(\mathbf{u}_{h}\right)^{2}\right)^{\frac{1}{2}}
$$

where $R\left(\mathbf{u}_{h}\right)$ is called residuum error and $\operatorname{NCF}\left(\mathbf{u}_{h}\right)$ is a measure of the nonconformity error.

Denoting by $X:=H^{2}\left(\Omega, \mathscr{T}_{K}\right)=\left\{\phi: \phi \in H^{2}(K), \forall K \in \mathscr{T}_{K}\right\}$ the broken Sobolev space, these quantities are defined as:

$$
\begin{gathered}
R\left(\mathbf{u}_{h}\right):=\left\|\mathscr{N}_{h}\left(\mathbf{u}_{h}, \cdot\right)\right\|_{X}=\sup _{\phi \in X \backslash\{0\}} \frac{\left\|\mathscr{N}_{h}\left(\mathbf{u}_{h}, \phi\right)\right\|}{\|\phi\|_{X}} \\
\operatorname{NCF}_{K}\left(\mathbf{u}_{h}\right):=\int_{\partial K \in \mathscr{E}_{i}} h_{\partial K}^{-1}\left[\mathbf{u}_{h}\right]^{2} \mathrm{~d} S+\int_{\partial K \in \mathscr{E}_{b}} h_{\partial K}^{-1}\left(\mathbf{u}_{h}-\mathbf{u}_{b}\right)^{2} \mathrm{~d} S \quad \operatorname{NCF}\left(\mathbf{u}_{h}\right):=\left(\sum_{K \in \mathscr{T}_{K}} \operatorname{NCF}_{K}\left(\mathbf{u}_{h}\right)^{2}\right)^{\frac{1}{2}}
\end{gathered}
$$

For the analytical solution we have that $\mathscr{N}_{h}(\mathbf{u}, \phi)=0, \forall \phi \in X$, while for the numerical solution this is true only for $\phi \in S_{h}^{p}$, thus the residuum error is a measure of the residual in the weak form of Eq. (1).

Using Eq. (29) and Eq. (20), it can be readily shown that

$$
\left|J(\mathbf{u})-J\left(\mathbf{u}_{h}\right)\right|=\left|\mathscr{N}_{h}\left(\mathbf{u}_{h}, z\right)\right| \leq R\left(\mathbf{u}_{h}\right)\|z\|_{X}
$$

Therefore, limiting the residuum error has a similar effect to limiting the residual-based indicator defined by Eq. (27) in the evaluation of the error in a target functional. The residuum error cannot be exactly computed, as it is defined on an infinite-dimensional space. Thus, the residuum error estimator and the local or element residuum error estimator are used in practice and are defined as:

$$
\begin{gathered}
\rho_{h}\left(\mathbf{u}_{h}\right):=\sup _{\phi \in S_{h}^{p+1} \backslash\{0\}} \frac{\left\|\mathscr{N}_{h}\left(\mathbf{u}_{h}, \phi\right)\right\|}{\|\phi\|_{X}} \\
\rho_{h, K}\left(\mathbf{u}_{h}\right):=\sup _{\phi \in S_{K}^{p+1} \backslash\{0\}} \frac{\left\|\mathscr{N}_{h}\left(\mathbf{u}_{h}, \phi\right)\right\|}{\|\phi\|_{X}}
\end{gathered}
$$

The spaces $S_{h}^{p+1}$ and $S_{K}^{p+1}$ correspond respectively to the spaces of piecewise polynomials of maximum degree $p+1$ and its restriction to the considered element for the evaluation of the element residuum estimator, defined by:

$$
S_{K}^{p+1}:=\left\{\phi \in L^{2}(K):\left.\phi\right|_{K} \in \mathscr{P}^{p+1}(K),\left.\phi\right|_{K^{\prime}}=0, \forall K, K^{\prime} \in \mathscr{T}_{k}, K \neq K^{\prime}\right\}
$$

In this work, following Dolejšri ${ }^{47}$ we consider the norm

$$
\|\cdot\|_{X}:=\left(\|\cdot\|_{L^{2}(\Omega)}^{2}+\frac{1}{\operatorname{Re}}|\cdot|_{H^{1}\left(\Omega, T_{K}\right)}^{2}\right)^{\frac{1}{2}}
$$

where Re is the Reynolds number. Other norms have also been employed in the literature. ${ }^{48,49}$ It can be shown ${ }^{27}$ that, based on this choice, the residuum error estimator can be directly computed from the element-wise values:

$$
\rho_{h}\left(\mathbf{u}_{h}\right)^{2}=\sum_{K \in \mathscr{T}_{K}} \rho_{h, K}\left(\mathbf{u}_{h}\right)^{2}
$$


The local residuum estimator $\rho_{h, K}\left(\mathbf{u}_{h}\right)$ is computed by seeking a solution $\psi=\sum_{i=1}^{N} \xi_{i} \phi_{i}$, where $\left\{\phi_{i}\right\}$ is a basis for $S_{K}^{p+1}$ and $\xi_{i}$ are the unknown coefficients, such that $\left|\mathscr{N}_{h}\left(\mathbf{u}_{h}, \psi\right)\right|$ is maximum and $\|\psi\|_{X}=1$. This leads to a constrained optimisation problem which can be solved via the Lagrange multipliers techniques, which requires the computation of the residuals $\mathscr{N}_{h}\left(\mathbf{u}_{h}, \phi_{i}\right)$.

It should be noted that $\mathscr{N}_{h}\left(\mathbf{u}_{h}, \psi\right), \forall \phi_{i} \in S_{K}^{p}$ should be identically null by virtue of the Galerkin orthogonality property. In practice this is not the case due to the presence of an algebraic error which is the combination of the aliasing and iterative errors. Thus, in order to evaluate this contribution, we compute the quantity $\mathscr{N}_{h}\left(\mathbf{u}_{h}, \psi\right)$ by increasing the number of overintegration points.

In conclusion, we indicate as residuum-NCF refinement indicator :

$$
\eta_{K}:=\left(\rho_{h, K}\left(\mathbf{u}_{h}\right)^{2}+\mathrm{NCF}_{K}\left(\mathbf{u}_{h}\right)^{2}\right)^{\frac{1}{2}}
$$

\section{Evaluation of the performances of refinement indicators}

In this section the results from the application of the adaptive $p$-refinement methodology based on the indicators described above are presented. Firstly, we consider the inviscid flow over a Gaussian bump at Mach number $\mathrm{M}=0.5$ and the inviscid flow past a cylinder at $\mathrm{M}=0.3$. Secondly, we analyse the laminar flow past a cylinder at $\mathrm{M}=0.1$ and Reynolds number $\mathrm{Re}=40$, based on the upstream velocity and the cylinder diameter. These configurations are subsonic and are characterised by solid boundaries free of geometrical singularities. For this reason, they are suitable for adaptive $p$-refinement. In fact, configurations presenting geometrical singularities or shock discontinuities are best treated by a combination of $h$ and $p$ refinement.

\section{IV.A. Steady Euler flow over a Gaussian Bump}

The first test case considered is the inviscid flow over a Gaussian bump at $\mathrm{M}=0.5$. This test case has been widely studied at the series of International Workshops of High-Order CFD Methods. ${ }^{7}$ The configuration is 2D with a subsonic inlet boundary condition, a prescribed static pressure at the outlet boundary and slip conditions imposed at the upper and bottom walls. The mesh employed is a $4^{\text {th }}$-order mesh which is generated based on the analytical expression of the bump that folows a Gaussian profile

The performance of the refinement indicators will be assessed by comparing the entropy error on the full domain, namely:

$$
\|e\|_{L^{2}(\Omega)}^{2}=\int_{\Omega}\left(\frac{p}{p_{\infty}}\left(\frac{\rho_{\infty}}{\rho}\right)^{\gamma}-1\right)^{2} \mathrm{~d} \Omega
$$

where $p_{\infty}$ and $\rho_{\infty}$ are the static pressure and density corresponding to inlet conditions.

Figure 1 shows the variation of this error measure versus the number of DOFs when uniform $p$-refinement is performed (solid black line with circles) as well as for the locally $p$-adapted simulations. For the latter, we report the results based on the refinement indicators introduced in the previous section. All the adaptive simulations have been performed starting from uniform $p=1$ and limiting the maximum local degree to $p=4$.

The global refinement indicator $\eta_{\text {glob }}$ is here defined as the entropy error given in Eq. (38) and the threshold value is set equal to a value slightly higher than that corresponding to uniform $p=4$. Figure 2 illustrates the refinement levels obtained at the end of the refinement procedure.

As reference, we also report the results obtained by using the local entropy error as refinement indicator (light blue dots in Fig. 1). For inviscid subsonic flows with uniform inlet conditions, this quantity can be considered as an exact measure of the discretization error. Therefore, these results illustrate one of the possible drawbacks of DEbased refinement indicators as mentioned in section I. In fact, for this configuration it is expected that a higher spatial resolution will be required in the proximity of the bump. In underresolved simulations an error in the entropy will be produced in this region and it will be convected downstream. For this reason, as can be seen in Fig. 2, this refinement indicator will effectively select for further refinement the region behind the bump. This will cause the suboptimal performance demonstrated in Fig. 1.

With regards to the other refinement indicators, we can see from Fig. 1 that they all show similar results, in spite of their fairly different derivation, implementation, and computational cost. The residual-based, residuum-NCF and NCF refinement indicators show slightly better results after the initial refinement step with the residual-based being the best performing indicator. The residual-based indicator reduces the number of DOFs necessary to achieve the same convergence level as uniform $p=4$ by $\sim 63 \%$ while the VMS and spectral decay indicator lead to a reduction of $\sim 58 \%$. 


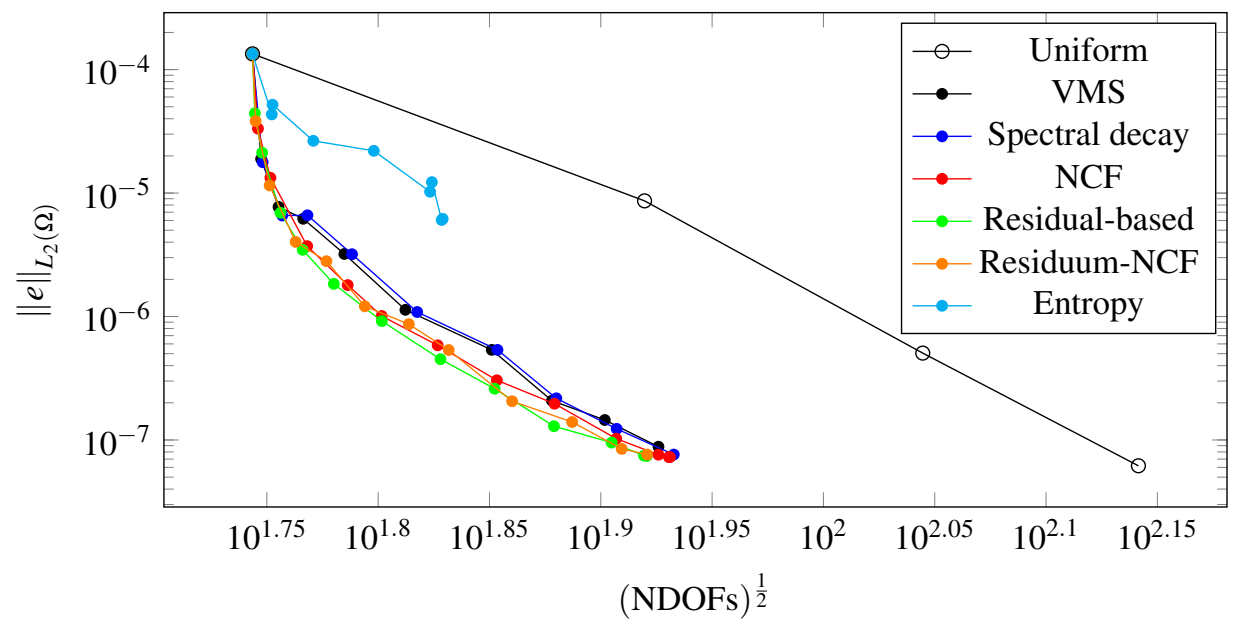

Figure 1: Euler flow over a Gaussian bump at $\mathrm{M}=0.5$ : evolution of global entropy error Eq. (38) under uniform and adaptive refinement.

It is also interesting to note that the VMS and NCF indicators, despite being DE estimators, do not suffer from the drawbacks illustrated for the local entropy error indicator.

Coming back to Fig. 2 it can be observed that the residual-based, NCF and residuum-NCF indicators exhibit fairly similar $p$-refinement patterns while those of the VMS and spectral decay indicators are nearly identical. As expected, the residuum-NCF indicator produces a refinement pattern which is in between the ones produced by the residual-based and the NCF-based estimators. Finally we point out that the NCF indicator generates a checkerboard-like pattern. This is despite the smooth distribution of the error indicator computed from the starting $p$-uniform solution.

\section{IV.B. Steady Euler flow past a cylinder}

The second test case considered is the inviscid flow past a cylinder at Mach number 0.3. Slip boundary conditions are imposed on the wall and non-reflecting boundary conditions are specified on the external boundary. The mesh employed is a O-type $4^{\text {th }}$-order mesh generated using Gmsh. ${ }^{50}$ This mesh has a radius $R=400 D$, with a number of elements $N_{r}=20$ and $N_{\theta}=26$ in the radial and azimuthal directions respectively. In the radial direction, the element size changes following a geometric progression of ratio 1.2 up to $r=4 D$, and 1.6 up to the domain boundary. As in the previous test case the $p$-adaptive algorithm is applied by starting from uniform $p=1$ and limiting the maximum local degree to 4 .

Due to the fact that a geometric progression is employed in the radial direction, there is a significant variation in element size for the employed mesh.

In this case, the application of the VMS and residual-based indicators leads to extremely poor results, selecting for refinement the largest elements even in the presence of low average values of the error. This is possibly caused by the direct dependency to the element size in Eq. (14) and Eq. (27). This is not the case for the other considered indicators which all employ some form of normalization. This is the reason why we consider in the following the normalized version of the VMS indicator given in Eq. (14) :

$$
\eta_{K}:=\left(\frac{\int_{K}\left\|(\rho \mathbf{v})_{h, p}-(\rho \mathbf{v})_{h, p-1}\right\|^{2} \mathrm{~d} V}{|K|}\right)^{\frac{1}{2}}
$$

where $|K|$ is the volume of the element $K$ and the residual-based indicator is also normalized by dividing Eq. (27) by the characteristic size of the element $h_{K}$ :

$$
\eta_{K}:=\left\|\mathbf{R}\left(\mathbf{u}_{h}\right)\right\|_{L^{2}(K)}+h_{K}^{-\frac{1}{2}}\left\|\mathbf{r}\left(\mathbf{u}_{h}\right)\right\|_{L^{2}\left(\partial K \cap \mathscr{E}_{i}\right)}+h_{K}^{-\frac{1}{2}}\left\|\mathbf{r}_{b}\left(\mathbf{u}_{h}\right)\right\|_{L^{2}\left(\partial K \cap \mathscr{E}_{b}\right)}
$$

Figure 3 reports the entropy error corresponding to uniform and adaptive $p$-refinement. As in the previous test case, all refinement indicators provide improved performances with respect to uniform refinement. The number of 


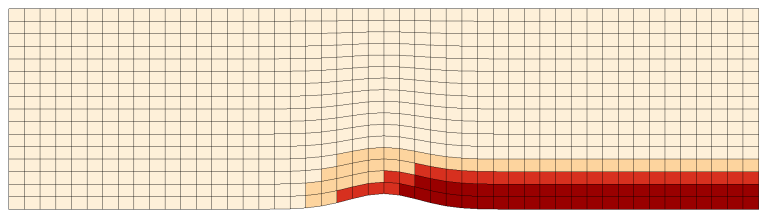

(a) Local entropy error indicator

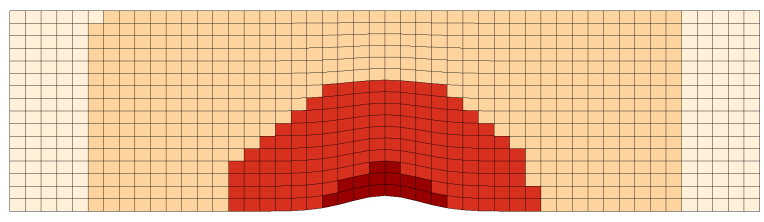

(c) Spectral decay indicator

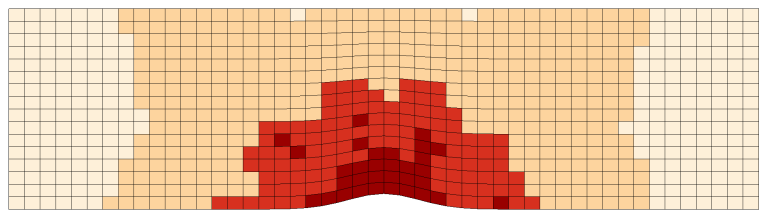

(e) Residual-based indicator

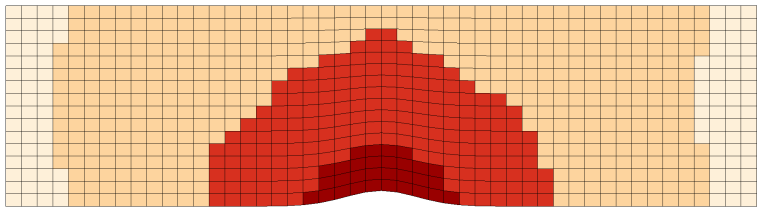

(b) VMS indicator

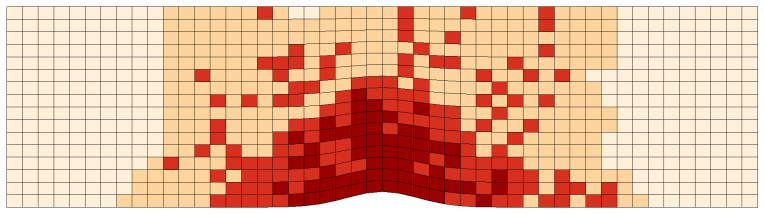

(d) NCF indicator

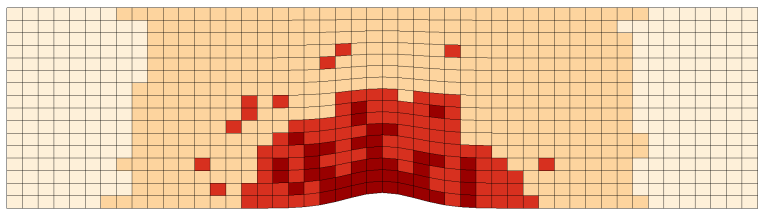

(f) Residuum-NCF indicator

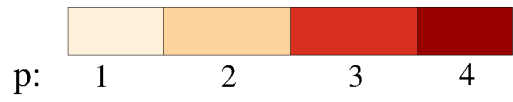

Figure 2: Euler flow over a Gaussian bump at $\mathrm{M}=0.5$ : map of local polynomial degrees obtained based on different refinement indicators.

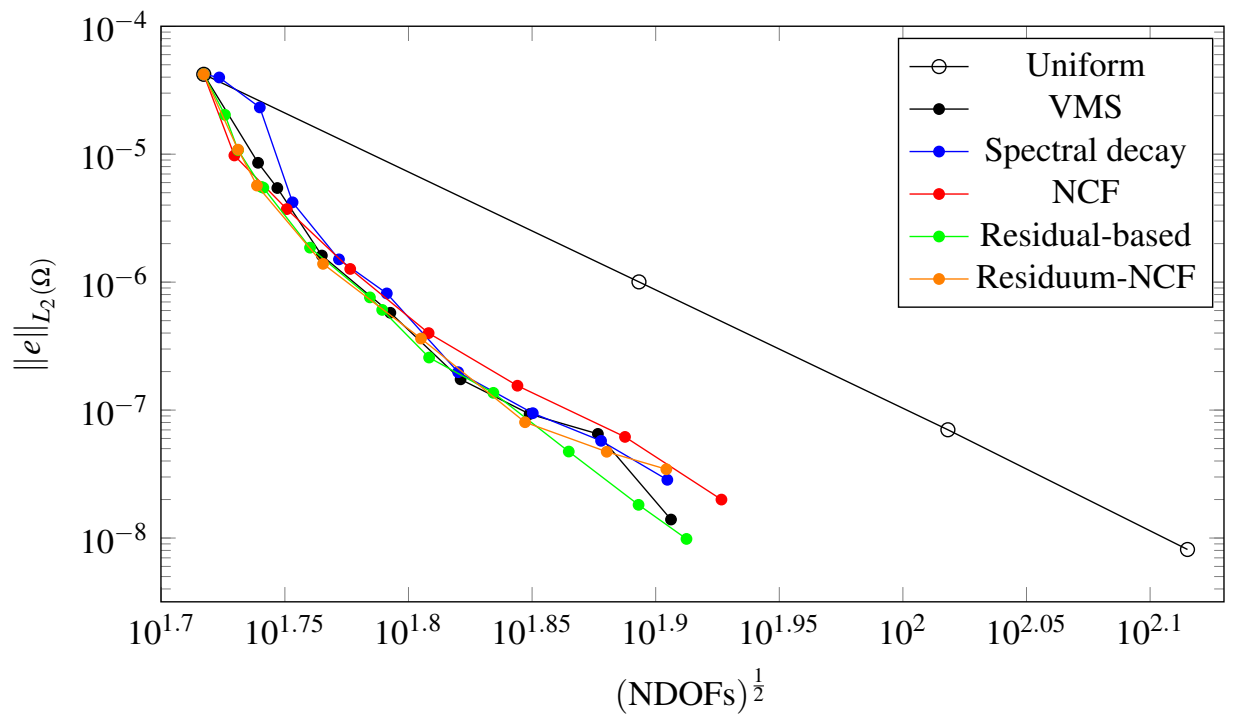

Figure 3: Euler flow past a cylinder at $\mathrm{M}=0.3$ : evolution of global entropy error Eq. (38) under uniform and adaptive refinement. 


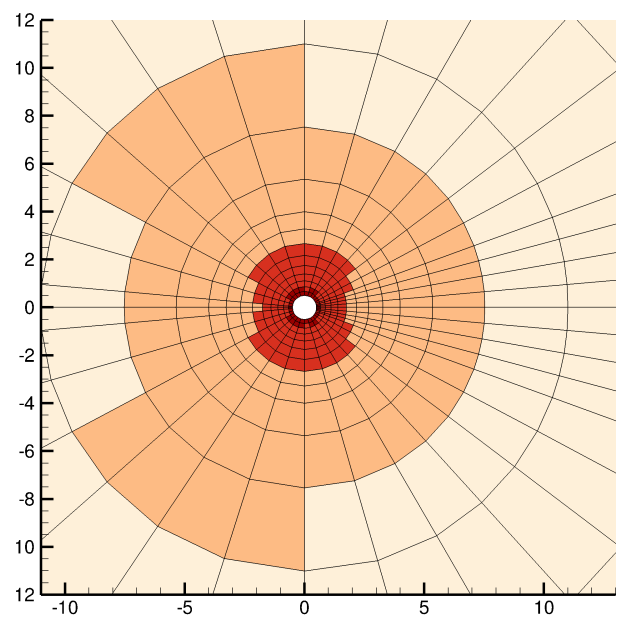

(a) VMS indicator

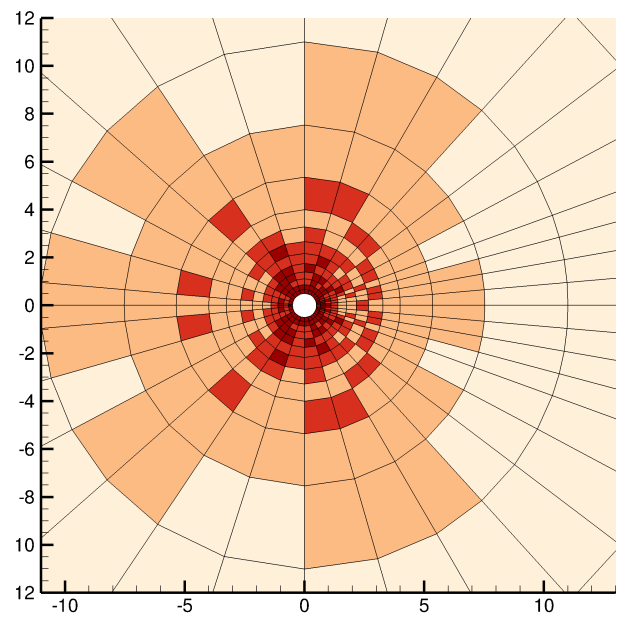

(c) NCF indicator

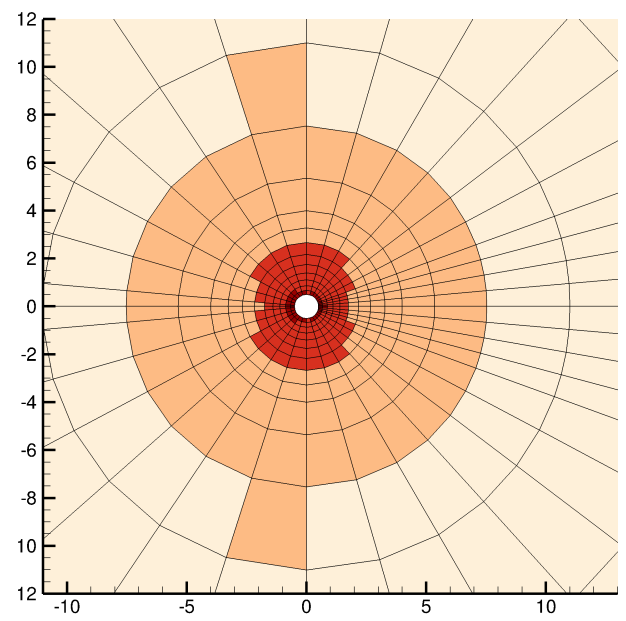

(b) Spectral decay indicator

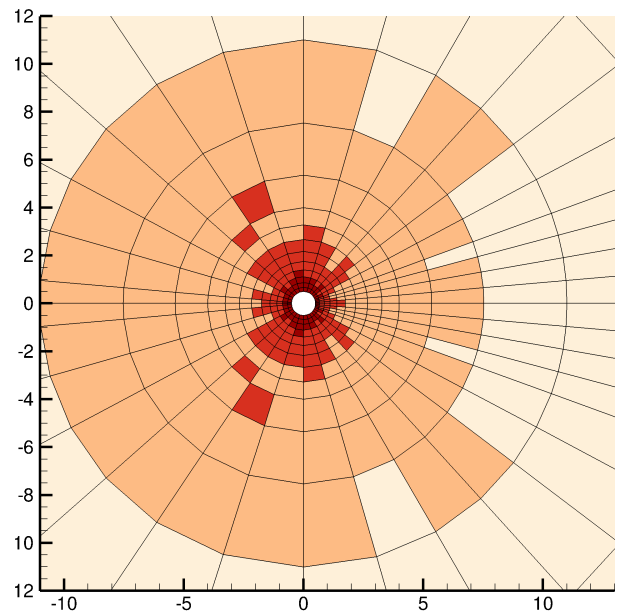

(d) Residual-based indicator
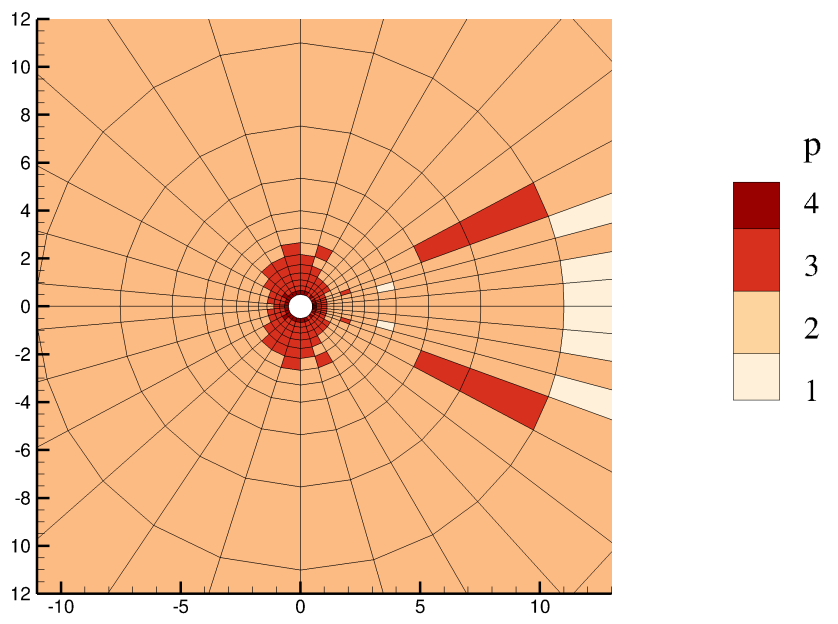

(e) Residuum-NCF indicator

Figure 4: Euler flow past a cylinder at $\mathrm{M}=0.3$ : map of local polynomial degrees obtained based on different refinement indicators . 
DOFs necessary to achieve the same accuracy as uniform $p=4$ is reduced by $\sim 60 \%$. Differences can be observed for the last steps of the refinement process, with the NCF indicator yielding the poorest results.

Figure 4 shows the distribution of the local polynomial degree across the domain obtained using the different refinement indicators. As in the previous section, we observe that the VMS and the spectral decay indicators provide very similar refinement patterns. As regards the NCF indicator, the checkerboard-like pattern observed in the previous test case (Fig. 2) is also visible here. Interestingly compared to the residual-based estimator, the residuum-NCF indicator refines over a larger region around the cylinder before increasing the local $p$ values in its proximity. This is possibly the cause of the slight loss in performance observed in Fig. 3 at high number of DOFs.

\section{IV.C. Steady laminar flow past a cylinder at $\mathrm{Re}=\mathbf{4 0}$}

Finally, we consider the laminar flow past a circular cylinder at $\mathrm{Re}=40$ and $\mathrm{M}=0.1$. For the considered Reynolds number the flow field is two-dimensional, symmetric and steady, thus allowing for computationally inexpensive comparisons on a laminar flow configuration. The flow pattern presents separation leading to the formation of two recirculation bubbles in the near wake that need to be correctly resolved in order to compute the drag coefficient accurately. This is the reason why this configuration is often employed for the validation of CFD codes.

Two-dimensional simulations are therefore performed by imposing no-slip boundary conditions at the wall and non-reflecting boundary conditions on the external boundary. The accuracy of the solution will mainly depend on three parameters: the spatial resolution, the extension of the computational domain and the accuracy in the representation of the cylinder geometry.

It has been shown that the size of the computational domain for unbounded flows is of critical importance as it is responsible for blockage effects and can distort phenomena generated in the internal domain convected through the artificial boundary. ${ }^{51,52}$ A computational domain of radius $R=2000 D$ is therefore employed here and has been verified to have an effect on the drag coefficient in the order of $\mathcal{O}\left(10^{-5}\right)$.

The mesh employed for the following simulations is a O-type $4^{\text {th }}$-order mesh with $N_{r}=18$ and $N_{\theta}=28$ elements in the radial and azimuthal direction respectively. In the radial direction the element size changes following a geometric progression of ratio 1.25 up to $r=6 D$ and 1.65 up to the external boundary.

In order to reduce the effect of quadrature errors, all the simulations where performed by using a number of integration points $q=p+5$ in each spatial direction. We have verified that, increasing this value has negligible effects on the obtained solutions.

For the simulations presented in this section, the adaptive $p$-refinement has been performed by starting from uniform polynomial degree $p=2$ and limiting the maximum local polynomial degree to $p=6$. By limiting the maximum polynomial degree to $p=6$ we can expect the solution of adaptive refinement to reach a level of accuracy similar to that obtained from uniformly refined simulation at $p=6$, albeit for a reduced number of DOFs. Figures 5 and 6 show the evolution of the error in the drag coefficient and in the $L^{2}$-norm using uniform and adaptive $p$-refinement with respect to a reference solution obtained with uniform $p=7\left(C_{D_{\text {ref }}}=1.50219\right)$. As the uniform $p=6$ solution produces an error in the drag coefficient of the order of $10^{-5}$, any further reduction of this error with $p$-adaptive refinement can be explained as small oscillations around the $C_{D}$ value obtained from the uniform $p=6$ solution.

All refinement indicators are able to reduce by $\sim 75 \%$ the number of DOFs necessary to achieve the same accuracy as that provided by uniform $p=6$.

Only minor differences can be observed between different refinement indicators in Fig. 5 and 6. If we consider the error in the drag coefficient, we cab observe from Fig. 5b that the residual-based and residuum-NCF refinement indicators show slightly better performance. As regards the discretization error in Fig. 6, the residuum-NCF refinement indicator and the VMS estimator appear to lead to the fastest convergence, with the residuum-NCF indicator performing best. However, after the initial steps, similar results are produced overall by the other refinement indicators.

Figures 7 and 8 show the distribution of local $p$-refinement levels corresponding to the last iteration of the adaptive refinement procedure for different estimators. Note that, as the maximum marking criterion was employed, the number of additional DOFs introduced at each refinement iteration could not be controlled. Therefore, the results presented correspond to a similar but not exactly the same number of DOFs and number of iterations. While some differences can be observed, the distributions of local polynomial degree are fairly similar for all the considered refinement indicators. All the indicators appear to select for refinement an approximately circular region around the cylinder and the shear region extending up to the outer boundary (see Fig. 8). Moreover, all refinement indicators select for further refinement the regions right in front of the cylinder and those located at an angle of $90^{\circ}$, before the separation of the flow which is located at $125^{\circ} .{ }^{51}$

One of the main differences that can be observed is the disparity in the refinement levels obtained in the recirculation region and in proximity of the cylinder base. Among all refinement indicators, the VMS and spectral decay 


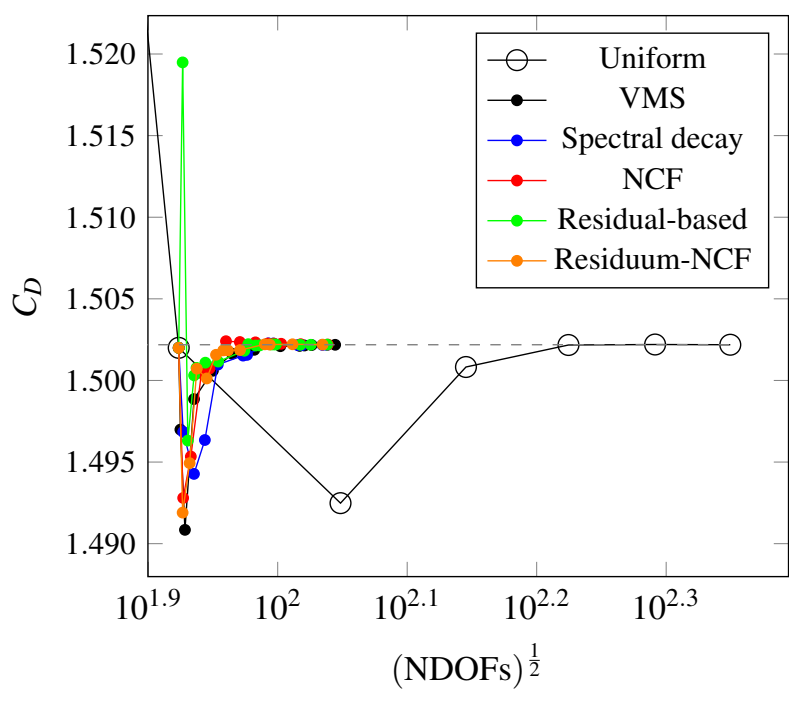

(a) Drag coefficient

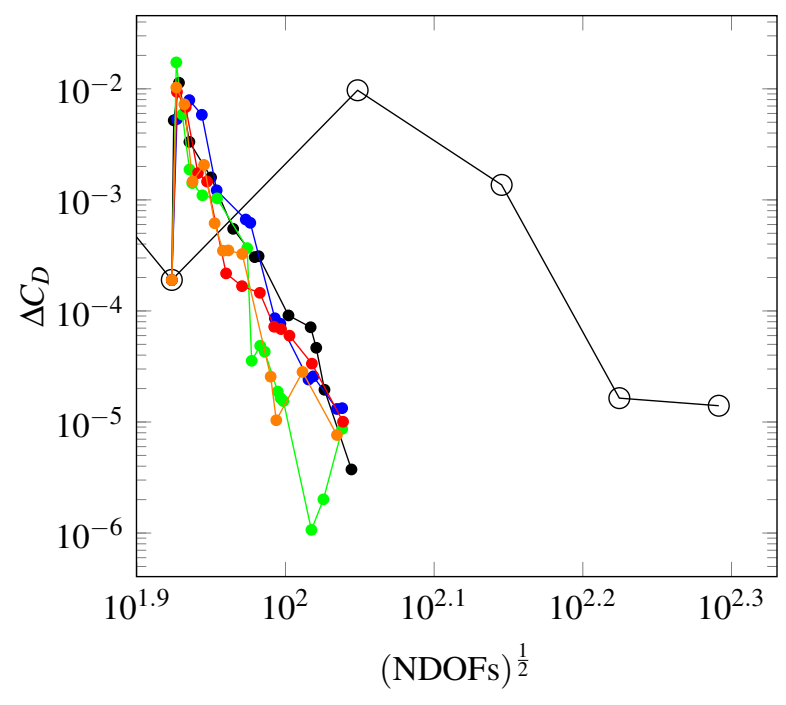

(b) Error in the drag coefficient

Figure 5: Evolution of the drag coefficient and corresponding error under uniform and adaptive $p$-refinement for the flow past a cylinder at $\operatorname{Re}=40$ and $M=0.1$.

indicators lead respectively to the lowest and highest refinement levels in this region. This difference in behaviour between the VMS and spectral decay indicators is somewhat expected. Indeed the spectral decay indicator normalizes the estimate of DE by the total 'energy' of the flow which means that it will tend to select for further refinement regions that are characterized at the same time by lower values of the error but also lower 'energy' (e. g. recirculation regions or stagnation points). This difference could be the cause of the slower convergence rate demonstrated by the spectral decay indicator in terms of the $L_{2}$-norm of the error in the momentum density.

In Fig. 8 the same refinement levels are shown for the far-wake region. As already observed, all refinement indicators select for refinement the far-wake region extending to the outer boundary. This is possibly because the large size of the elements requires an increase in polynomial degree in order to correctly capture the behaviour of the flow in this region. Therefore, this refinement reduces the $L_{2}$-norm of the error in the momentum density even if it has a limited effect on the convergence of the drag coefficient.

Finally we note that the checkerboard-like pattern shown for the NCF indicator, in the Euler case, is not observed in Fig. 7 although it has been observed in the intermediate steps of the refinement process.

\section{Implementation issues and computational cost}

In section IV, the performances of the different refinement indicators have been analysed in terms of the reduction in the number of DOFs yielded by each estimator. It is indeed difficult to compare the computational time required by each approach due to the presence of several parameters that might affect the results obtained and therefore the derived conclusions. Firstly, we have observed that the choice of the marking parameter $\theta$ has a significant effect on the total computational time, while limited changes where observed in terms of the evolution of the error versus the number of DOFs of the adaptive refinement procedure. This is caused by the direct link between the computational time and the number of refinement steps which in turn depends on the marking strategy employed. Moreover, the computational cost of the refinement indicators strongly depends on the details of the implementation. Finally, direct comparison of the computational times for distributed memory computations will also be influenced by the possibility of achieving a good load balance by taking into account the local polynomial degree $p$.

In this section, we make some general observations that might be useful even when considering a different implementation from the one employed in our work. In particular, we consider the locality properties of the refinement indicators and their possible effect on parallel efficiency, the ease of implementation in existing CFD codes and the number of operations required by each indicator.

As regards locality, we can see from the definition of the different refinement indicators provided in section III that, the only two indicators which are fully local are the VMS and spectral decay indicators as their computation (see. Eq. (14) and Eq. (16)) only requires the knowledge of the solution inside the element. On the other hand, the 


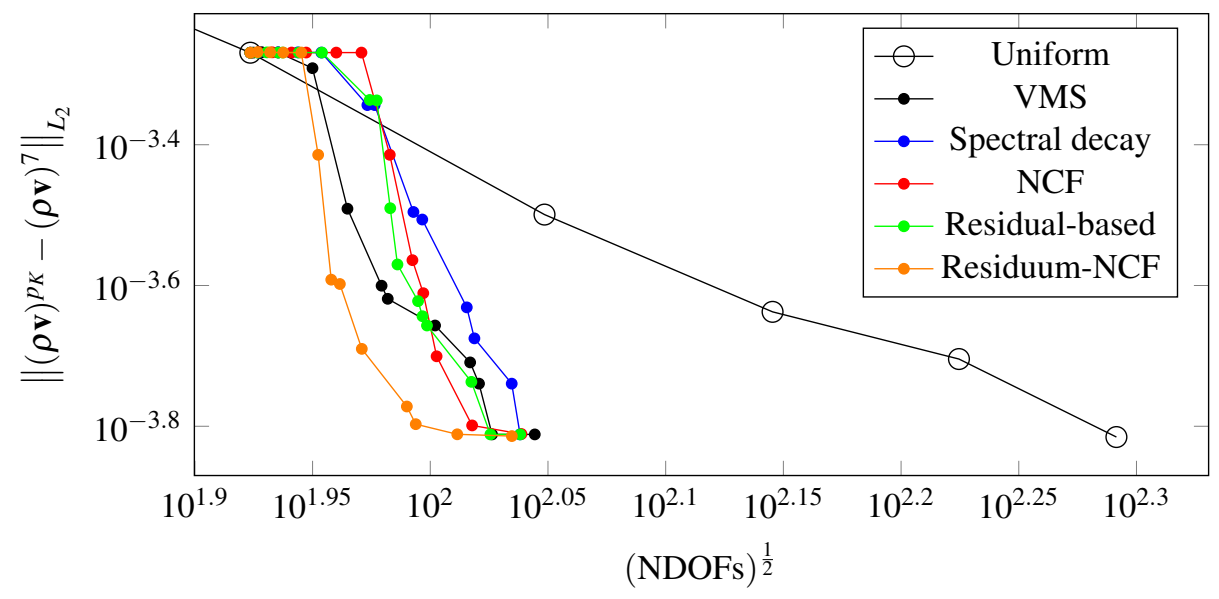

Figure 6: $L^{2}$-norm of the error in the momentum density under uniform and adaptive $p$-refinement for the flow past a cylinder at $\mathrm{Re}=40$ and $\mathrm{M}=0.1$.

computation of the NCF indicator (given in Eq. (18)) requires knowledge of the projection at the interface of the solution from the direct neighbours, while the residual-based and the residuum-NCF indicators will require additional information to be exchanged for the computation of the numerical fluxes. In the framework of distributed memory computations this lack of locality will involve blocking message passing operations which may lead to a reduced parallel efficiency.

Considering now the number of operations involved in the computation of each indicator, we observe that the VMS and spectral decay indicators require the projection of the solution on the reduced order space $S_{h}^{p-1}$ and the computation of the integrals to compute the $L_{2}$-norm. In the case of a modal DG approach based on hierarchical basis functions, the scale separation is readily available and the required integrals can be efficiently computed by employing information contained in the mass matrix (see Tumolo et al. ${ }^{41}$ ). On the other hand, if a non-hierarchical basis is employed (e. g. nodal approach), the additional projection operation and the integration by quadrature will lead to higher computational cost. In this particular case, the NCF indicator would be more computationally efficient as it only requires the interpolation of the solution at the gauss points on each interface. As regards the residual-based and residuum-NCF indicators, their computation is more expensive as compared to the other indicators. Indeed, the evaluation of the former requires the computation and integration over the elements and faces of the element residuals, numerical fluxes and the convective and diffusive fluxes obtained from the internal reconstruction $\mathscr{F}\left(\mathbf{u}_{h}^{+}, \nabla \mathbf{u}_{h}^{+}\right)$. Finally, we expect the residuum-NCF to be the most expensive among the considered indicators, regardless of the particular CFD solver considered. This is because, the contribution of the nonconformity error is more expensive to compute than the NCF indicator alone as it requires the integration of the jump terms that appear in the expression of the NCF indicator. The evaluation of the residuum-error contribution, on the other hand, requires the computation of the discrete residuals $\mathscr{N}_{h}\left(\mathbf{u}_{h}, \phi_{i}\right)$ with $\phi_{i}$ being the basis functions corresponding to the space $S_{h}^{p+1}$. To this we have to add the cost of the Lagrange multiplier method involved in the computation of this indicator which requires the solution of a linear system of size $\left(p_{k}+2\right)^{d}$ for each element.

Considering now the ease of implementation, it is interesting to note that the complexity of computing the residuals corresponding to the refined polynomial space $S_{h}^{p+1}$ will depend on the numerical implementation of the CFD method. In the case of the Aghora solver, ${ }^{32}$ this required the computation and storage of the basis functions for $S_{h}^{p+1}$ as well as their derivatives on the corresponding set of quadrature points. In general, important modifications of the employed CFD solver might be required to achieve a computationally efficient implementation of this method. This is not the case for the VMS, spectral decay and NCF indicators which can be easily implemented even as external post-processing tools. On the other hand, the definition of the different terms involved in the definition of the residual-based indicator (Eq. (22), Eq. (23) and Eq. (24)) depends on the set of equations being solved and the discretization employed.

These observations might be of limited interest in the context of steady-flow simulations, since the computation of a steady solution is likely to be more time consuming than the refinement procedure. However, these considerations will be of primary importance when extending these techniques to unsteady-flow simulations for which the refinement procedure is carried out over the course of the simulation. 


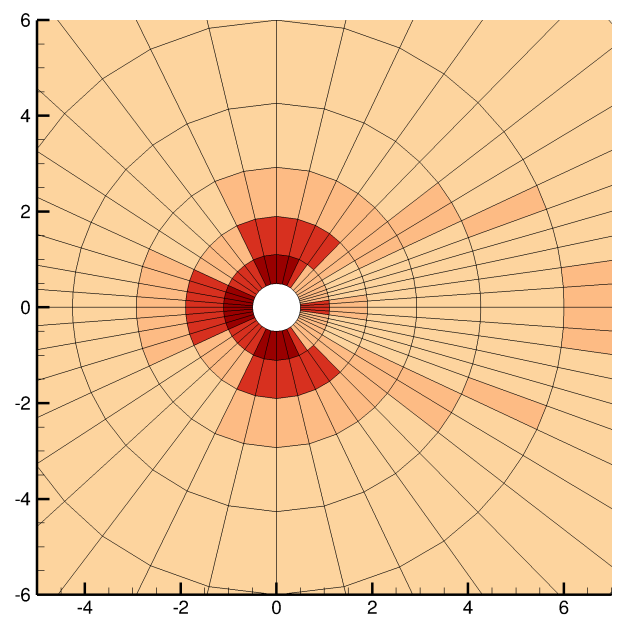

(a) VMS indicator

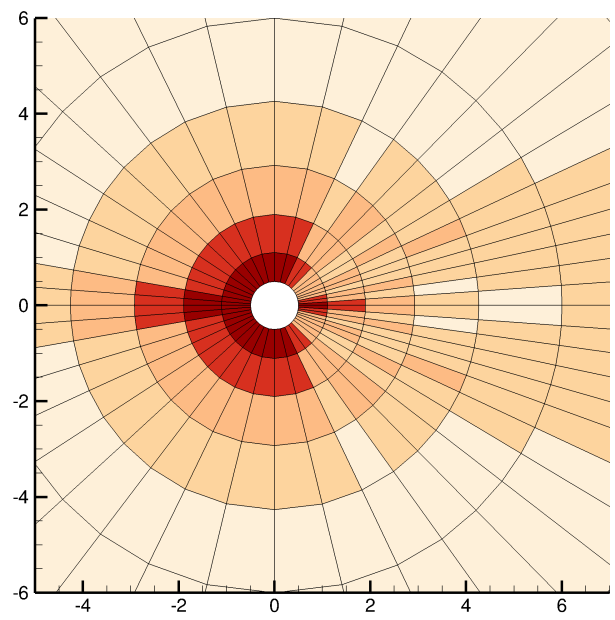

(c) NCF indicator

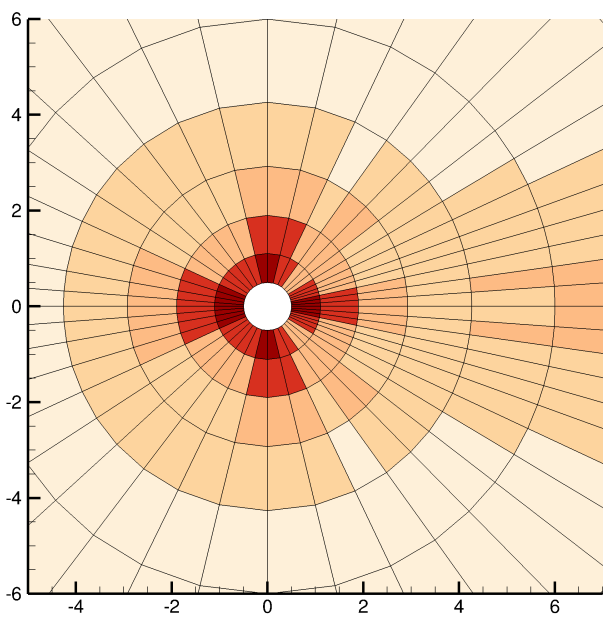

(b) Spectral decay indicator

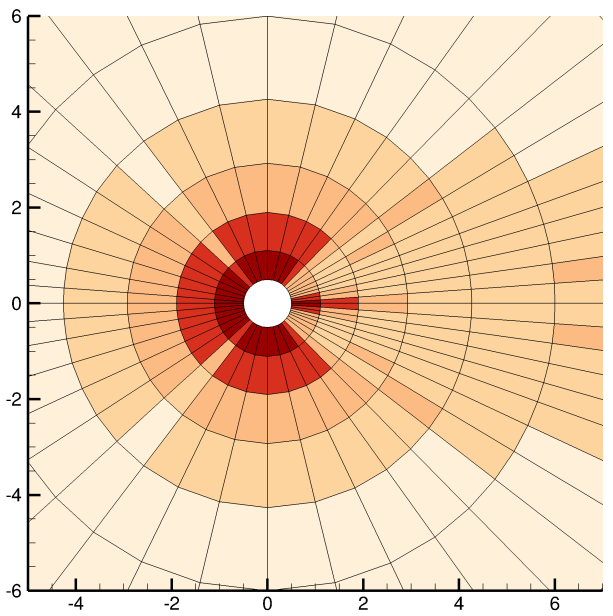

(d) Residual-based indicator
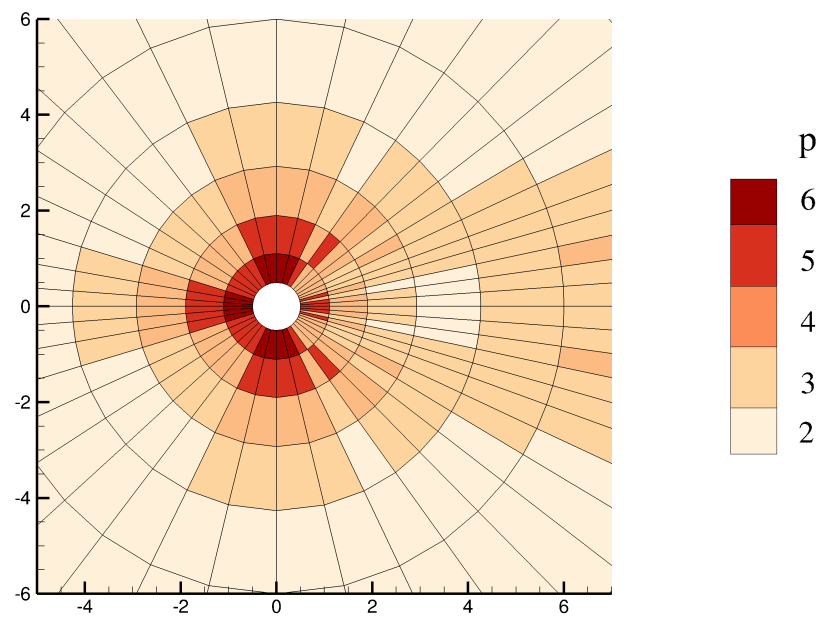

(e) Residuum-NCF indicator

Figure 7: Local polynomial orders obtained with different refinement indicators for the laminar flow past a cylinder at $\operatorname{Re}=40$ and $M=0.1$. 


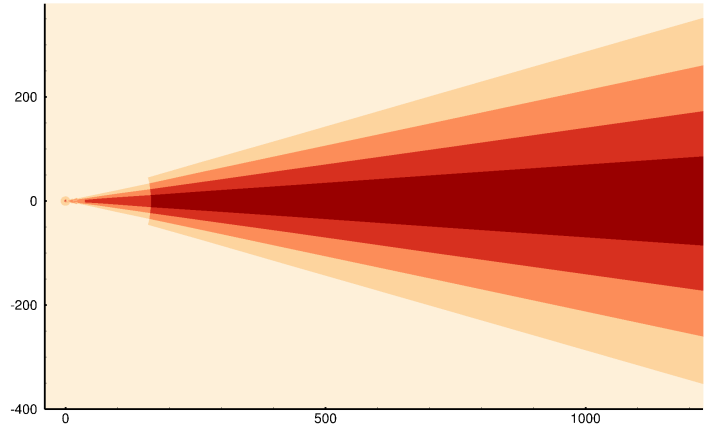

(a) VMS indicator

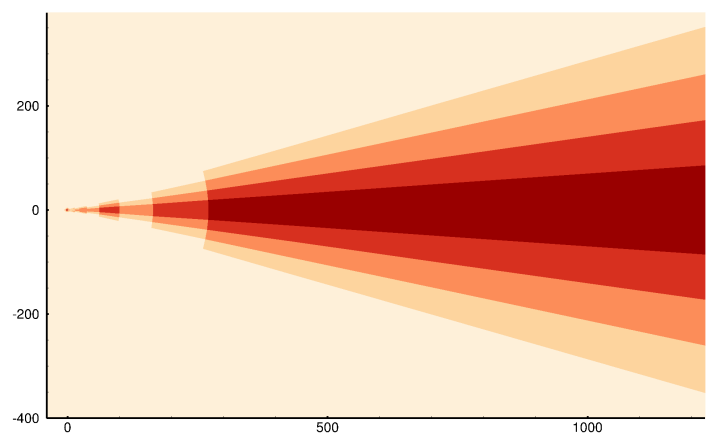

(c) NCF indicator

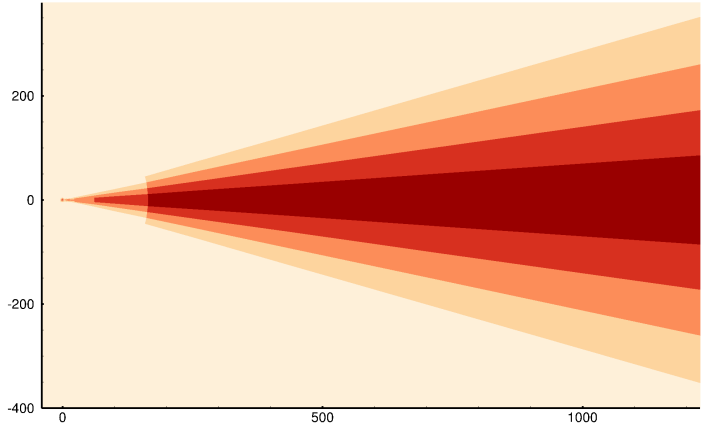

(b) Spectral decay indicator

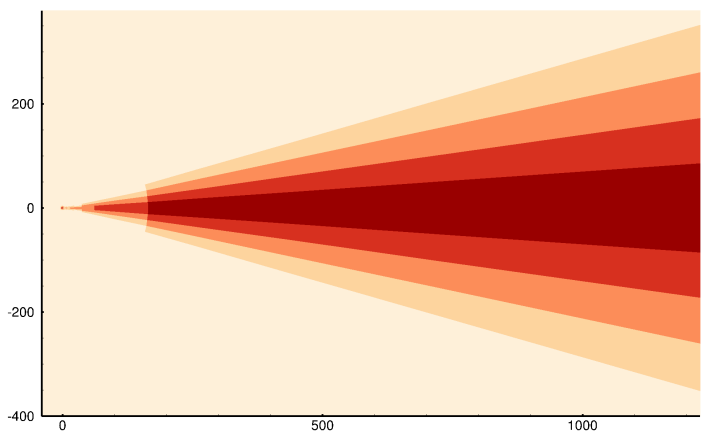

(d) Residual-based indicator

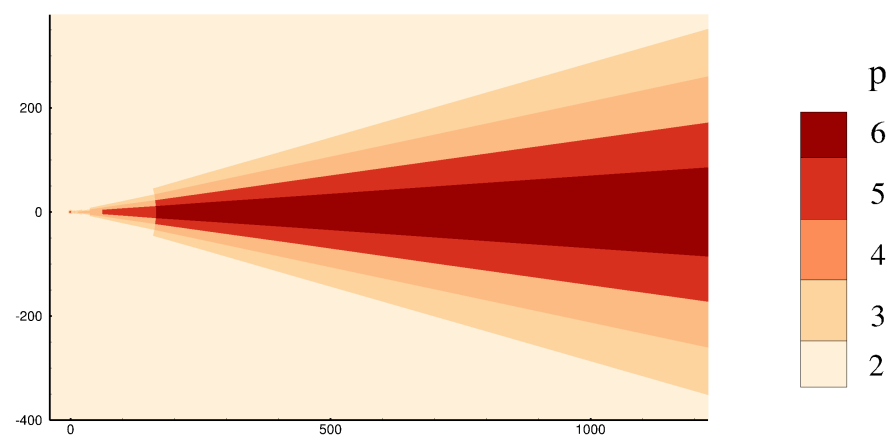

(e) Residuum-NCF indicator

Figure 8: Local polynomial orders obtained with different refinement indicators for the laminar flow past a cylinder at $\operatorname{Re}=40$ and $M=0.1$. 


\section{Conclusion}

In this article we have assessed the performance of various refinement indicators for the implementation of an adaptive $p$-refinement algorithm in the context of a DG method. Five refinement indicators of different types, namely, feature-based, discretization and residual error-based have been employed for the simulation of inviscid and laminar flow configurations. The choice of the error estimators considered in this work has been made based on their potential suitability for the simulation of turbulent flows by LES. The goal-oriented approach to error estimation has therefore been discarded due to its high computational cost when dealing with unsteady flow simulations.

Three flow configurations have been considered: the inviscid flow over a Gaussian bump at $\mathrm{M}=0.5$, the inviscid flow past a circular cylinder at $M=0.3$ and the laminar flow past a circular cylinder at $M=0.1$ and $R e=40$. The advantage of adaptive refinement as compared to uniform refinement in terms of number of DOFs has been clearly observed. Indeed, for all the configurations considered, the number of degrees of freedom necessary to achieve a prescribed level of accuracy was reduced by $60 \%$ to $75 \%$ thanks to the use of the $p$-adaptive algorithm.

Minor advantages with respect to other estimators have been observed from the application of the residual-based indicator to Euler flow simulations and from the application of the residuum-NCF indicator to the laminar flow simulations. However, similar results have been observed for the VMS, spectral decay and NCF indicators both in terms of the refinement pattern and convergence history. This is interesting if we consider the lack in their formulation of any specific terms related to the set of equations being solved and the discretization being employed. Moreover these estimators can be easily implemented as post-processing tools and require a reduced computational cost as we have seen from section V. In particular, the VMS and spectral decay indicators can be evaluated with very limited computational cost when a modal DG method based on hierarchical basis functions is employed. In contrast the computation of the residual-based and residuum-NCF indicators might require a considerable effort in order to obtain an efficient implementation well adapted to the particular solver at hand.

With regards to the VMS and spectral decay indicators, the former demonstrated similar if not superior performances as compared to the latter in each case considered. An advantage of the VMS over the spectral decay indicator is its lower computational cost regardless of the implementation as the normalization term at the denominator of Eq. (16) does not need to be computed.

Finally we have observed that the NCF indicator generates a checkerboard-like pattern which appears to depend on the refinement history. This pattern could potentially damage the convergence of the method as it introduces an irregularity in the spatial discretization.

All these results are of interest as they provide guidelines for the application of $p$-refinement to unsteady flow simulations. Indeed, for such applications, the computational cost of the refinement indicators will be a fundamental parameter in the choice of the adaptive refinement strategy. Other important issues that must be considered when designing adaptive refinement methods for unsteady flow computations will include the definition of a suitable marking criterion, refinement and derefinement thresholds and the possibility for the user to define relevant values for these $a$ priori. The extension of this comparison to $h$ and $h p$-refinement is the subject of current research.

\section{Acknowledgments}

This project has received funding from the European Union's Horizon 2020 research and innovation programme under the Marie Skłodowska-Curie grant agreement No 675008. This work was performed using HPC resources from GENCI (Grant 2017-A0022A10129). We also thank Mathieu Lorteau, Emeric Martin, Florent Renac and Marie-Claire Le Pape for the valuable discussions.

\section{References}

\footnotetext{
${ }^{1}$ Abbà, A., Bonaventura, L., Nini, M., and Restelli, M., "Dynamic models for Large Eddy Simulation of compressible flows with a high order DG method," Computers \& Fluids, Vol. 122, 2015, pp. 209-222.

${ }^{2}$ Kuru, G., de la Llave Plata, M., Couaillier, V., Abgrall, R., and Coquel, F., "An adaptive Variational Multiscale Discontinuous Galerkin Method For Large Eddy Simulation," 54th AIAA Aerospace Sciences Meeting, San Diego, California, AIAA, 2016.

${ }^{3}$ Lesaint, P. and Raviart, P. A., "On a finite element method for solving the neutron transport equation," Publications mathématiques et informatique de Rennes, Vol. S4, 1974, pp. 1-40.

${ }^{4}$ Reed, W. H. and Hill, T. R., "Triangular mesh methods for the neutron transport equation," Los Alamos Report LA-UR-73-479, 1973.

${ }^{5}$ Cockburn, B. and Shu, C.-W., "TVB Runge-Kutta local projection discontinuous Galerkin finite element method for conservation laws. II. General framework," Mathematics of computation, Vol. 52, No. 186, 1989, pp. 411-435.

${ }^{6}$ Cockburn, B. and Shu, C.-W., "Runge-Kutta discontinuous Galerkin methods for convection-dominated problems," Journal of scientific computing, Vol. 16, No. 3, 2001, pp. 173-261.
} 
${ }^{7}$ Wang, Z. J., Fidkowski, K., Abgrall, R., Bassi, F., Caraeni, D., Cary, A., Deconinck, H., Hartmann, R., Hillewaert, K., Huynh, H. T., et al., "High-order CFD methods: current status and perspective," International Journal for Numerical Methods in Fluids, Vol. 72, No. 8, 2013, pp. 811-845.

${ }^{8}$ Kroll, N., Bieler, H., Deconinck, H., Couaillier, V., van der Ven, H., and Sorensen, K., ADIGMA-A European Initiative on the development of adaptive higher-order variational methods for aerospace applications: results of a collaborative research project funded by the European Union, 2006-2009, Vol. 113, Springer Science \& Business Media, 2010.

${ }^{9}$ Biswas, R., Devine, K. D., and Flaherty, J. E., "Parallel adaptive finite element methods for conservation laws," Applied Numerical Mathematics, Vol. 14, No. 1-3, 1994, pp. 255-283.

${ }^{10}$ Chapelier, J.-B., de la Llave Plata, M., Renac, F., and Lamballais, E., "Evaluation of a high-order discontinuous Galerkin method for the DNS of turbulent flows," Computers \& Fluids, Vol. 95, 2014, pp. 210-226.

${ }^{11}$ de la Llave Plata, M., Couaillier, V., and le Pape, M.-C., "On the use of a high-order discontinuous Galerkin method for DNS and LES of wall-bounded turbulence," Computers \& Fluids, 2017.

${ }^{12}$ Roy, C. J., "Review of discretization error estimators in scientific computing," AIAA Paper, Vol. 126, 2010, pp. 2010

${ }^{13}$ Houston, P. and Süli, E., "A note on the design of hp-adaptive finite element methods for elliptic partial differential equations," Computer Methods in Applied Mechanics and Engineering, Vol. 194, No. 2, 2005, pp. 229-243.

${ }^{14}$ Mitchell, W. F. and McClain, M. A., "A comparison of hp-adaptive strategies for elliptic partial differential equations," ACM Transactions on Mathematical Software (TOMS), Vol. 41, No. 1, 2014, pp. 2.

${ }^{15}$ Mitran, S. M., “A comparison of adaptive mesh refinement approaches for Large-Eddy Simulation,” Tech. rep., DTIC Document, 2001.

${ }^{16}$ Wackers, J., Deng, G., Guilmineau, E., Leroyer, A., Queutey, P., and Visonneau, M., "Combined refinement criteria for anisotropic grid refinement in free-surface flow simulation," Computers \& Fluids, Vol. 92, 2014, pp. 209-222.

${ }^{17}$ Oden, J. T., Wu, W., and Legat, V., "An hp adaptive strategy for finite element approximations of the Navier-Stokes equations," International journal for numerical methods in fluids, Vol. 20, No. 8-9, 1995, pp. 831-851.

${ }^{18}$ Dolejší, V. and Solin, P., "hp-discontinuous Galerkin method based on local higher order reconstruction," Applied Mathematics and Computation, Vol. 279, 2016, pp. 219-235.

${ }^{19}$ Mavriplis, C., "Adaptive mesh strategies for the spectral element method," Computer methods in applied mechanics and engineering, Vol. 116, No. 1, 1994, pp. 77-86.

${ }^{20}$ Creusé, E. and Nicaise, S., "A posteriori error estimator based on gradient recovery by averaging for convection-diffusion-reaction problems approximated by discontinuous Galerkin methods," IMA Journal of Numerical Analysis, Vol. 33, No. 1, 2013, pp. 212-241.

${ }^{21}$ Banks, J., Hittinger, J., Connors, J., and Woodward, C., "Numerical error estimation for nonlinear hyperbolic PDEs via nonlinear error transport," Computer Methods in Applied Mechanics and Engineering, Vol. 213, 2012, pp. 1-15.

${ }^{22}$ Syrakos, A., Efthimiou, G., Bartzis, J. G., and Goulas, A., "Numerical experiments on the efficiency of local grid refinement based on truncation error estimates," Journal of Computational Physics, Vol. 231, No. 20, 2012, pp. 6725-6753.

${ }^{23}$ Kompenhans, M., Rubio, G., Ferrer, E., and Valero, E., "Adaptation strategies for high order discontinuous Galerkin methods based on Tau-estimation," Journal of Computational Physics, Vol. 306, 2016, pp. 216-236.

${ }^{24}$ Hartmann, R. and Houston, P., "Adaptive discontinuous Galerkin finite element methods for the compressible Euler equations," Journal of Computational Physics, Vol. 183, No. 2, 2002, pp. 508-532.

${ }^{25}$ Dolejší, V., Ern, A., and Vohralík, M., "A framework for robust a posteriori error control in unsteady nonlinear advection-diffusion problems," SIAM Journal on Numerical Analysis, Vol. 51, No. 2, 2013, pp. 773-793.

${ }^{26}$ Riviere, B. and Wheeler, M. F., "A posteriori error estimates for a discontinuous Galerkin method applied to elliptic problems. Log number: R74," Computers \& Mathematics with Applications, Vol. 46, No. 1, 2003, pp. 141-163.

${ }^{27}$ Dolejší, V., Roskovec, F., and Vlasák, M., "Residual based error estimates for the space-time discontinuous Galerkin method applied to the compressible flows," Computers \& Fluids, Vol. 117, 2015, pp. 304-324.

${ }^{28}$ Fidkowski, K. J. and Darmofal, D. L., "Review of output-based error estimation and mesh adaptation in computational fluid dynamics," AIAA journal, Vol. 49, No. 4, 2011, pp. 673-694.

${ }^{29}$ Hartmann, R., "Multitarget error estimation and adaptivity in aerodynamic flow simulations," SIAM Journal on Scientific Computing, Vol. 31, No. 1, 2008, pp. 708-731.

${ }^{30}$ Antepara, O., Lehmkuhl, O., Borrell, R., Chiva, J., and Oliva, A., "Parallel adaptive mesh refinement for Large-Eddy Simulations of turbulent flows," Computers \& Fluids, Vol. 110, 2015, pp. 48-61.

${ }^{31}$ Tugnoli, M., Abbà, A., Bonaventura, L., and Restelli, M., "A locally p-adaptive approach for Large Eddy Simulation of compressible flows in a DG framework," Journal of Computational Physics, Vol. 349, November 2017, pp. 33-58.

${ }^{32}$ Renac, F., de la Llave Plata, M., Martin, E., Chapelier, J.-B., and Couaillier, V., "Aghora: a high-order DG solver for turbulent flow simulations," IDIHOM: Industrialization of High-Order Methods-A Top-Down Approach, Springer, 2015, pp. 315-335.

${ }^{33}$ Botti, L., "Influence of reference-to-physical frame mappings on approximation properties of discontinuous piecewise polynomial spaces," Journal of Scientific Computing, Vol. 52, No. 3, 2012, pp. 675-703.

${ }^{34}$ Bassi, F., Rebay, S., Mariotti, G., Pedinotti, S., and Savini, M., "A high-order accurate discontinuous finite element method for inviscid and viscous turbomachinery flows," Proceedings of the 2nd European Conference on Turbomachinery Fluid Dynamics and Thermodynamics, Antwerpen, Belgium, 1997, pp. 99-109.

${ }^{35}$ Bassi, F., Crivellini, A., Rebay, S., and Savini, M., "Discontinuous Galerkin solution of the Reynolds-averaged Navier-Stokes and k- $\omega$ turbulence model equations," Computers \& Fluids, Vol. 34, No. 4, 2005, pp. 507-540.

${ }^{36}$ Hughes, T. J., Mazzei, L., and Jansen, K. E., "Large Eddy Simulation and the variational multiscale method," Computing and visualization in science, Vol. 3, No. 1, 2000, pp. 47-59.

${ }^{37}$ Mavriplis, C., "A posteriori error estimators for adaptive spectral element techniques," Proceedings of the Eighth GAMM-Conference on Numerical Methods in Fluid Mechanics, Springer, 1990, pp. 333-342.

${ }^{38}$ Mavriplis, C., Nonconforming discretizations and a posteriori error estimators for adaptive spectral element techniques, Ph.D. thesis, Massachusetts Institute of Technology, 1989. 
${ }^{39}$ Persson, P.-O. and Peraire, J., "Sub-cell shock capturing for discontinuous Galerkin methods," 44th AIAA Aerospace Sciences Meeting and Exhibit, 2006, p. 112.

${ }^{40}$ Gassner, G., Altmann, C., Hindenlang, F., Staudenmeier, M., and Munz, C., "Explicit discontinuous Galerkin schemes with adaptation in space and time," 36th CFD/ADIGMA course on hp-adaptive and hp-multigrid methods, VKI LS, 2009.

${ }^{41}$ Tumolo, G., Bonaventura, L., and Restelli, M., "A semi-implicit, semi-Lagrangian, p-adaptive discontinuous Galerkin method for the shallow water equations," Journal of Computational Physics, Vol. 232, No. 1, 2013, pp. $46-67$.

${ }^{42}$ Tumolo, G. and Bonaventura, L., "A semi-implicit, semi-Lagrangian discontinuous Galerkin framework for adaptive numerical weather prediction," Quarterly Journal of the Royal Meteorological Society, Vol. 141, No. 692, 2015, pp. 2582-2601.

${ }^{43}$ Krivodonova, L. and Flaherty, J. E., "Error estimation for discontinuous Galerkin solutions of two-dimensional hyperbolic problems," Advances in Computational Mathematics, Vol. 19, No. 1-3, 2003, pp. 57-71.

${ }^{44}$ Krivodonova, L., Xin, J., Remacle, J.-F., Chevaugeon, N., and Flaherty, J. E., "Shock detection and limiting with discontinuous Galerkin methods for hyperbolic conservation laws," Applied Numerical Mathematics, Vol. 48, No. 3-4, 2004, pp. 323-338.

${ }^{45}$ Ainsworth, M., "A posteriori error estimation for Discontinuous Galerkin finite element approximation," SIAM Journal on Numerical Analysis, Vol. 45, No. 4, 2007, pp. 1777-1798.

${ }^{46}$ Hartmann, R., Held, J., Leicht, T., and Prill, F., "Error estimation and adaptive mesh refinement for aerodynamic flows," ADIGMA-A European Initiative on the Development of Adaptive Higher-Order Variational Methods for Aerospace Applications, Springer, 2010, pp. 339-353. 118.

${ }^{47}$ Dolejší, V., "hp-DGFEM for nonlinear convection-diffusion problems," Mathematics and Computers in Simulation, Vol. 87, 2013, pp. 87-

${ }^{48}$ Dolejší, V. and Roskovec, F., "Residual based error estimates for the space-time discontinuous Galerkin method applied to nonlinear hyperbolic equations," Proceedings of the Conference Algoritmy, 2016, pp. 113-124.

${ }^{49}$ Dolejší, V., "A design of residual error estimates for a high order BDF-DGFE method applied to compressible flows," International Journal for Numerical Methods in Fluids, Vol. 73, No. 6, 2013, pp. 523-559.

${ }^{50}$ Geuzaine, C. and Remacle, J.-F., "Gmsh: A 3-D finite element mesh generator with built-in pre-and post-processing facilities," International Journal for Numerical Methods in Engineering, Vol. 79, No. 11, 2009, pp. 1309-1331.

${ }^{51}$ Gautier, R., Biau, D., and Lamballais, E., "A reference solution of the flow over a circular cylinder at Re=40," Computers \& Fluids, Vol. 75, 2013, pp. 103-111.

${ }^{52}$ Posdziech, O. and Grundmann, R., "A systematic approach to the numerical calculation of fundamental quantities of the two-dimensional flow over a circular cylinder," Journal of Fluids and Structures, Vol. 23, No. 3, 2007, pp. 479-499. 